\title{
ON A CLASS OF EDGE-TRANSITIVE DISTANCE-REGULAR ANTIPODAL COVERS OF COMPLETE GRAPHS ${ }^{1}$
}

\author{
Ludmila Yu. Tsiovkina \\ Krasovskii Institute of Mathematics and Mechanics, \\ Ural Branch of the Russian Academy of Sciences, \\ 16 S. Kovalevskaya Str., Ekaterinburg, 620108, Russia \\ tsiovkina@imm.uran.ru
}

\begin{abstract}
The paper is devoted to the problem of classification of edge-transitive distance-regular antipodal covers of complete graphs. This extends the classification of those covers that are arc-transitive, which has been settled except for some tricky cases that remain to be considered, including the case of covers satisfying condition $c_{2}=1$ (which means that every two vertices at distance 2 have exactly one common neighbour).

Here it is shown that an edge-transitive distance-regular antipodal cover of a complete graph with $c_{2}=1$ is either the second neighbourhood of a vertex in a Moore graph of valency 3 or 7, or a Mathon graph, or a half-transitive graph whose automorphism group induces an affine 2-homogeneous group on the set of its fibres. Moreover, distance-regular antipodal covers of complete graphs with $c_{2}=1$ that admit an automorphism group acting 2-homogeneously on the set of fibres (which turns out to be an approximation of the property of edge-transitivity of such cover) are described.

A well-known correspondence between distance-regular antipodal covers of complete graphs with $c_{2}=1$ and geodetic graphs of diameter two that can be viewed as underlying graphs of certain Moore geometries, allows us to effectively restrict admissible automorphism groups of covers under consideration by combining Kantor's classification of involutory automorphisms of these geometries together with the classification of finite 2-homogeneous permutation groups.
\end{abstract}

Keywords: Distance-regular graph, Antipodal cover, Geodetic graph, Arc-transitive graph, Edge-transitive graph, 2-transitive group, 2-homogeneous group.

\section{Introduction}

A distance-regular antipodal cover of a complete graph can be defined as a connected graph whose vertex set admits a partition into $n$ classes (called fibres) of the same size $r \geq 2$ such that each class induces a coclique, the union of any two distinct classes induces a perfect matching, and any two non-adjacent vertices from distinct classes have exactly $c_{2} \geq 1$ common neighbours. According to [8], such a graph will be referred to as an $\left(n, r, c_{2}\right)$-cover. One can see that an $\left(n, r, c_{2}\right)$-cover is indeed a cover (or a covering graph) of the complete graph $K_{n}$ in the topological sense (see [8] or [7]), and that its diameter is 3 .

To date, almost all arc-transitive ( $\left.n, r, c_{2}\right)$-covers have been classified (see [14, 15, 19-22]), except for the following two tricky cases: when an arc-transitive automorphism group induces an affine permutation group on the set of fibres (see [22]) or $c_{2}=1$ (see a discussion below in this section). Note that an arc- or, more generally, edge-transitive automorphism group of an $\left(n, r, c_{2}\right)$-cover induces a 2-homogeneous action on its fibres. The purpose of this paper is to study the $(n, r, 1)$ covers whose automorphism group acts 2-homogeneously on the set of fibres, and to describe those that are edge-transitive.

\footnotetext{
${ }^{1}$ This work was supported by the Russian Science Foundation under grant no. 20-71-00122.
} 
The afore-mentioned interplay between edge-transitivity and 2-homogeneity allows us to base our arguments on the classification of finite 2-homogeneous permutation groups, which follows from the classification of finite 2-transitive permutation groups and the Kantor's fundamental result [11]. To investigate admissible groups of automorphisms, we also exploit a remarkable correspondence between $(n, r, 1)$-covers and geodetic graphs of diameter two (see [2]) that are equivalent to certain Moore geometries. The classification of involutory automorphisms of these geometries that is due to Kantor [12] together with the Higman's technique for studying automorphisms of association schemes (e.g., see [4, Section 3.7]) turn out to be effective tools for their description.

Main results of this paper are presented by the following two theorems.

Theorem 1. Let $\Delta$ be a $(k+1, r, 1)$-cover with $s:=k-r+1>1$, let $\Sigma$ be the set of fibres of $\Delta$, and $G=\operatorname{Aut}(\Delta)$. Denote by $K$ and $G^{\Sigma}$ the kernel and the image of the induced action of $G$ on $\Sigma$, respectively. Then $k=c s$ and $r=c s-s+1$ for some $c \in \mathbb{Z}$, and the following statements hold:

(1) if $G^{\Sigma}$ is a 2-homogeneous, but not 2-transitive group, then

$$
G^{\Sigma} \leq \operatorname{A\Gamma L}_{1}(q), \quad k+1=q \equiv 3 \quad(\bmod 4), \quad \sqrt{c s+(s / 2-1)^{2}} \notin \mathbb{Z},
$$

and either $K=1, s=2$ and $c=(q-1) / 2$, or $s$ is odd;

(2) if $G^{\Sigma}$ is an almost simple 2-transitive group, then either $K=1, s=2, c=2^{e-1}, \operatorname{Soc}(G) \simeq$ $\mathrm{L}_{2}\left(2^{e}\right)$ and $\Delta$ is a Mathon graph, or $G$ acts intransitively on vertices of $\Delta$;

(3) if $G^{\Sigma}$ is an affine 2-transitive group, then $G$ acts intransitively on arcs of $\Delta$.

Theorem 2. Suppose $\Delta$ is an edge-transitive $(k+1, r, 1)$-cover, let $\Sigma$ be the set of fibres of $\Delta$, and $G=\operatorname{Aut}(\Delta)$. Denote by $K$ and $G^{\Sigma}$ the kernel and the image of the induced action of $G$ on $\Sigma$, respectively. Then either $k=r \in\{2,6\}$ and $\Delta$ is the second neighbourhood of a vertex in a Moore graph of valency $k+1$, or $k>r$ and one of the following statements holds:

(1) $G^{\Sigma}$ is an almost simple 2-transitive group, $K=1$, $\operatorname{Soc}(G) \simeq \mathrm{L}_{2}\left(2^{e}\right)$ and $\Delta$ is an arc-transitive Mathon graph of valency $k=2^{e}$;

(2) $G^{\Sigma}$ is an affine 2-homogeneous group and $\Delta$ is a half-transitive graph.

Recall that the only Moore graphs of valency 3 or 7 are the Petersen graph or the HoffmanSingleton graph, respectively (see [10]). Note that for each admissible $k$ the resulting graph in Theorem 2 (1) is unique (up to isomorphism) and its construction is due to Mathon (e.g., see [3, Proposition 1.17.3]).

We also remark here that in [6, Proposition 4] it was claimed that each $(k+1, r, 1)$-cover with $s>1$ that possesses a group of automorphisms acting 2-homogeneously on the fibres necessarily has valency $k=2^{e}$ and $s=2$. Unfortunately, the proof of this result (see an exposition in [16]) is flawed; Theorem 1 shows that it holds under the additional assumption of arc-transitivity of the graphs under consideration. Thus, compared together with previous results (see [14, 15]), the classification of arc-transitive $\left(n, r, c_{2}\right)$-covers in the almost simple case is complete.

The organization of the paper is as follows. In Section 1 we recall some basic definitions and facts on $(n, r, 1)$-covers. In Section 2 we obtain general results on automorphisms of such a graph. Section 3 is devoted to the proofs of Theorems 1 and 2 . 


\section{Preliminaries}

Throughout the paper we consider only finite undirected graphs without loops or multiple edges. By a subgraph of a graph $\Gamma$ we mean a vertex-induced subgraph, and we also identify a subset $X$ of vertices of $\Gamma$ with the subgraph of $\Gamma$ that is induced by $X$. The distance between vertices $x$ and $y$ of a graph $\Gamma$ is denoted by $\partial_{\Gamma}(x, y)$, or simply $\partial(x, y)$ if $\Gamma$ is clear from the context. For a vertex $a$ of a graph $\Gamma$, we denote by $\Gamma_{i}(a)$ the $i$-th neighbourhood of $a$, that is the subgraph of $\Gamma$ induced by the set $\left\{b \in \Gamma \mid \partial_{\Gamma}(a, b)=i\right\}$. The number of neighbours of a vertex $a$, i.e., the size of $\Gamma_{1}(a)$, is the valency of $a$ in $\Gamma$. For a fixed graph $\Gamma$ and any its vertex $a$, the subgraph $\Gamma_{1}(a)$ is also denoted by $[a]$ if the graph $\Gamma$ is clear from the context; we also put $a^{\perp}:=\{a\} \cup[a]$. A graph is said to be regular if all its vertices have the same valency; a graph is said to be biregular if it is not regular and every of its vertices has one of two possible valencies.

A graph is geodetic if every two of its vertices are joined by a unique shortest path. A biregular geodetic graph of diameter two that is not contained in $a^{\perp}$ for any its vertex $a$ is referred to as a BRG-graph.

A connected graph $\Gamma$ of diameter $d$ is called distance-regular if there are integers $c_{i}, a_{i}$ and $b_{i}$, for all $i \in\{0,1, \ldots, d\}$, such that for each pair of vertices $x$ and $y$ with $\partial_{\Gamma}(x, y)=i$, the following equalities hold:

$$
c_{i}=\left|\Gamma_{i-1}(x) \cap \Gamma_{1}(y)\right|, \quad a_{i}=\left|\Gamma_{i}(x) \cap \Gamma_{1}(y)\right| \quad \text { and } \quad b_{i}=\left|\Gamma_{i+1}(x) \cap \Gamma_{1}(y)\right|,
$$

where $b_{d}=c_{0}=0$ by definition; in particular, $\left|\Gamma_{1}(x)\right|=b_{0}=c_{i}+a_{i}+b_{i}$ holds for any $i \in\{0,1, \ldots, d\}$. The sequence $\left\{b_{0}, b_{1}, \ldots, b_{d-1} ; c_{1}, \ldots, c_{d}\right\}$ is called the intersection array of $\Gamma$.

A distance-regular graph of diameter 2 is also called strongly regular. A graph is said to be edge regular if it is regular and there is a non-negative integer $\lambda$ such that every two adjacent vertices have exactly $\lambda$ common neighbours; a graph is said to be amply regular if it is edge-regular and there is a non-negative integer $\mu$ such that every two vertices at distance 2 have exactly $\mu$ common neighbours.

If the binary relation "to be at distance 0 or $d$ " on the set of vertices of a connected graph $\Gamma$ of diameter $d$ is an equivalence relation, then the graph $\Gamma$ is called antipodal; the classes of this relation are called antipodal classes or fibres of $\Gamma$. We will say that an antipodal graph $\Gamma$ is an antipodal cover of a graph $\Delta$, if $\Gamma$ is not a complete graph and the following three conditions are satisfied: $(i)$ every fibre of $\Gamma$ induces a coclique, $(i i)$ the union of any two distinct fibres of $\Gamma$ induces a coclique or a perfect matching, and (iii) $\Delta$ is isomorphic to the graph $\bar{\Gamma}$ defined on the fibres of $\Gamma$, in which two vertices are adjacent if and only if the union of corresponding fibres forms a matching in $\Gamma$. By the Smith's theorem [3, Theorem 4.2.1], non-cyclic distance-regular graphs fall into families of primitive, bipartite or antipodal graphs. Every graph of diameter $d$ from the latter family is a complete graph or a complete multipartite graph with parts of equal sizes if $d=1$ or 2 , and it is an antipodal cover of a distance-regular graph of diameter $\lfloor d / 2\rfloor$ when $d \geq 3[5]$. Hence distance-regular antipodal covers of complete graphs are precisely antipodal distance-regular graphs of diameter 3. They do not have a universal construction and form a large infinite class of graphs that is closely related to many interesting combinatorial objects, like projective planes or generalized quadrangles; we refer the reader to $[3,8,16]$ for more background.

For a subset $X$ of a group acting on a set $\Omega$, by $\operatorname{Fix}_{\Omega}(X)$ we denote the set of points in $\Omega$ that are fixed by every element of $X$. When $X=\{g\}$, we write "Fix $(g)$ " instead of "Fix $(\{g\})$ ". We also write $\operatorname{Fix}(X)=\operatorname{Fix}_{\Omega}(X)$ if $\Omega$ is clear from the context. In what follows, for a graph $\Gamma$ and a subset $X \subseteq \operatorname{Aut}(\Gamma)$, we identify the set $\operatorname{Fix}(X)$ with the subgraph of $\Gamma$ that is induced by $\operatorname{Fix}(X)$.

A graph is called vertex-transitive or edge-transitive, if its automorphism group acts transitively on the set of its vertices or on the set of its edges, respectively. A graph is called arc-transitive, if 
its automorphism group acts transitively on set of its arcs (ordered pairs of adjacent vertices). A graph is called half-transitive, if it is vertex- and edge-transitive, but not arc-transitive.

Our other terminology and notation are mostly standard and follow $[1,3]$.

Further in this section, we provide some auxiliary results that are used in the proofs of Theorems 1 and 2 .

Throughout the rest of the paper, $\Delta$ is an $(k+1, r, 1)$-cover, $\Sigma$ is the set of fibres of $\Delta$ and $s:=k-r+1$. By [8, Theorem 3.4] there is an integer $c$ such that $c s=k$, the number $c s+1$ is odd and $s \leq c$. Put

$$
v=(c s+1)(c s-s+1) \text { and } \quad D=c s-s+1+s^{2} / 4=c s+(s / 2-1)^{2} .
$$

Then $v$ is the number of vertices of $\Delta$ and its distinct eigenvalues are

$$
\theta_{0}=c s, \quad \theta_{1}=(s-2) / 2+\sqrt{D}, \quad \theta_{2}=-1, \quad \theta_{3}=(s-2) / 2-\sqrt{D}
$$

of respective multiplicities

$$
m_{0}=1, \quad m_{1}=\frac{(c s+1)(c s-s)}{2}\left(1-\frac{s-2}{2 \sqrt{D}}\right), \quad m_{2}=c s, \quad m_{3}=\frac{(c s+1)(c s-s)}{2}\left(1+\frac{s-2}{2 \sqrt{D}}\right) .
$$

Due to a result of Gardiner [5, Proposition 5.1] the eigenvalues of $\Delta$ are integral if $s \neq 2$. Hence for odd $s$ the number $2 \sqrt{D}$ is an odd integer (since $D=c s+1-s+s^{2} / 4$ ), while for even $s>2$ already the number $\sqrt{D}$ is an integer.

Let us construct a graph $\widehat{\Delta}$ by adding a coclique $A$ to $\Delta$, whose vertices are identified with the fibres of $\Delta$, together with a vertex $\hat{b}$ such that $\widehat{\Delta}(\hat{b})=A$, and assuming that a vertex $F \in A$ is adjacent to just those vertices of $\Delta$ which belong to the fibre $F \in \Sigma$. Note that each vertex from $A$ has valency $r+1$ in $\widehat{\Delta}$.

It is easy to see that $\widehat{\Delta}$ is a geodetic graph of diameter two and hence by [3, Theorem 1.17.1] either $\widehat{\Delta}$ is a strongly regular graph and $s=1$, or $\widehat{\Delta}$ is a BRG-graph with valencies $r+1$ and $k+1$, $r<k, s \geq 2$ and the following statements hold:

(1) if $A$ and $B$ denote the sets of vertices of $\widehat{\Delta}$ of valencies $r+1$ and $k+1$, respectively, then $A$ is a coclique, for each vertex $a \in A$ the subgraph $[a]$ is a coclique, and if $x$ and $y$ is a pair of adjacent vertices from $B$, then $|[x] \cap[y]|=k-r=s-1$;

(2) $|\widehat{\Delta}|=(r+1)(k+1)+1$.

Moreover, each geodetic graph of diameter two that has no vertex adjacent to all others, can be viewed as the underlying graph of a Moore geometry, i.e. an incidence system of points and lines which satisfies the following axioms:

(i) there is at least one line, and each line has at least two points;

(ii) two points are on at most one line;

(iii) no point is collinear with all others;

(iv) two non-collinear points are both collinear with exactly one common point;

$(v)$ a point not in a line is collinear with at most one point of the line;

(vi) there are no triangles or quadrangles of lines. 
Also, by [5, Proposition 5.2], if $s=1$, then $\widehat{\Delta}$ is a Moore graph (and $c \in\{2,6,56\}$ ). In what follows we assume that $s \geq 2$, so $\widehat{\Delta}$ is a BRG-graph, and its corresponding Moore geometry is said to have type $(c s+2, c s-s+3, s+1)$ in this case (see [12, p. 314]).

We say that $\widehat{\Delta}$ has type $\mathcal{D}_{\alpha}$, if there is a projective plane $(X, \mathcal{L})$ of order $\alpha=r+1$ with a polarity $\pi$ such that $\widehat{\Delta}$ is isomorphic to the graph on $X$, in which two vertices $x$ and $y$ are adjacent if and only if $x \in y^{\pi}$ (wherein $k=r+1$ and $A$ coincides with the set of absolute points of the polarity $\pi$ ).

Lemma 1. The following statements hold:

(1) $c \geq 2$, the number $c s+1$ is odd, $s \leq c$, and the neighbourhood of each vertex in $\Delta$ is the disjoint union of $c$ isolated cliques of size $s$;

(2) if $c=2$, then $\Delta$ is a unique distance-regular graph with intersection array $\{4,2,1 ; 1,1,4\}$ (the line graph of the Petersen graph) and $\widehat{\Delta}$ has type $\mathcal{D}_{4}$;

(3) if $2<c \leq 1000$, then either $s=2$ and $\widehat{\Delta}$ has type $\mathcal{D}_{2 c}$, or the pair $(s ; D)$ is one of: $(4 ; 25)$, $(4 ; 49),(3 ; 169 / 4),(6 ; 100),(9 ; 625 / 4),(4 ; 81),(11 ; 1225 / 4), 4 ; 121),(18 ; 784),(4 ; 169)$, $(35 ; 8649 / 4),(4 ; 225),(10 ; 676),(4 ; 289),(4 ; 361),(21 ; 7921 / 4),(46 ; 4900),(4 ; 441)$, $(11 ; 5625 / 4),(4 ; 529),(4 ; 625),(26 ; 4356),(14 ; 2500),(4 ; 729),(4 ; 841),(4 ; 961)$, $(4 ; 1089),(4 ; 1225),(8 ; 2601),(15 ; 20449 / 4),(4 ; 1369),(5 ; 7569 / 4),(4 ; 1521),(9 ; 14161 / 4)$, $(4 ; 1681),(152 ; 70225),(4 ; 1849),(4 ; 2025),(20 ; 10201),(4 ; 2209),(4 ; 2401),(144 ; 93025)$, $(4 ; 2601),(56 ; 38025),(44 ; 30625),(114 ; 81796),(4 ; 2809),(4 ; 3025),(4 ; 3249),(4 ; 3481)$, (7; 25281/4), (4;3721), (4;3969).

P r o o f. The first two statements follow by [3, Proposition 1.17.3] and [8, Theorem 3.4].

To prove the third statement, first observe that the number of cliques of size $s+1$ of $\Delta$ equals $(c s+1)(c s-s+1) c /(s+1)$. Then, for $2 \leq s \leq c \leq 1000$, the computer check in GAP (which uses integrality conditions for the eigenvalues of $\Delta$ and their multiplicities together with the condition of integrality of the number $(c s+1)(c s-s+1) c /(s+1))$ gives just those feasible pairs $(s ; D)$ that are listed in (3). The lemma is proved.

The above restrictions for parameters of $\Delta$ will be frequently used in following arguments, in particular, the list of feasible parameters from Lemma 1 (3) will be needed in Section 3 to rule out the existence of $\Delta$ in a series of special cases.

Lemma 2. Let $\Phi$ be an amply regular graph with $\mu=1$ and suppose there is an automorphism $g$ of $\Phi$ such that for a $\langle g\rangle$-orbit $\Psi$ each vertex $x \in \Psi$ is adjacent to $x^{g}$. Then $\Psi$ is a cycle or a clique.

P r o o f. Suppose $\Psi$ is not a clique. Denote by $i$ the least number in $\{2, \ldots,|\Psi|-1\}$ such that the vertices $x$ and $x^{g^{i}}$ are not adjacent. Then $\left\{x^{g}, x^{g^{i-1}}\right\} \subseteq[x] \cap\left[x^{g^{i}}\right]$ and hence $i=2$. Now let $j$ denote the least number in $\{3, \ldots,|\Psi|-1\}$ such that the vertices $x$ and $x^{g^{j}}$ are adjacent. Then $\left\{x^{g^{-1}}, x^{g^{j}}\right\} \subseteq[x] \cap\left[x^{g^{j-1}}\right]$ and hence $j=|\Psi|-1$. Thus, we conclude that $\Psi$ is a cycle.

The lemma is proved.

Recall that $(x, y)$ denotes the greatest common divisor of $x$ and $y$.

Lemma 3 [16, Lemma 2.2.1]. The graph $\Delta$ has exactly $c s+1$ fibres, each of size $c s-s+1$, and the following statements hold: 
(1) $s+1$ divides $c(c-1)(c-2)$ and each odd prime divisor of $D$ divides $(s-2, c)(4 c+1, s-4)\left(c-1, s^{2}+4\right)$;

(2) if $c>2$, then there is a divisor $d$ of cs such that $s=d(d-2) /(c-d)$ and $\sqrt{D}=(d+c(d-2) /(c-d)) / 2$, and if an odd prime $p$ divides $(D, s-2)$, then the p-part of $d$ is less than p-part of $c$;

(3) if $c s=2^{n}$, then $s=2$;

(4) if $s=2$, then $\widehat{\Delta}$ has type $\mathcal{D}_{2 c}$.

P r o o f. Note that $\Delta$ has exactly $c s+1$ fibres, each of size $c s-s+1$.

(1) Since there are exactly $c(c s+1)(c s-s+1) /(s+1)$ cliques of size $s+1$ in $\Delta, s+1$ divides $c(c-1)(c-2)$. Let $p$ be an odd prime divisor of $D$. Then $p$ divides $(c s+1)(c s-s)(s-2)$, $(p, c)=(p, s-2)$ and $(p, c s+1)=\left(p, s^{2} / 4-s\right)=(p, 4 c+1)$. So, we conclude $(p, c-1)=\left(p, 1+s^{2} / 4\right)$.

(2) Let $c>2$. Put $D=y^{2}$ and $y-s / 2+1=d$. Then

$$
y^{2}-(s / 2-1)^{2}=c s, \quad y+s / 2-1=c s / d .
$$

Further,

$$
y=(d+c s / d) / 2, \quad s / 2-1=(c s / d-d) / 2,
$$

hence

$$
s=d(d-2) /(c-d), \quad y=(d+c(d-2) /(c-d)) / 2 .
$$

Suppose an odd prime $p$ divides $(D, s-2)$. As

$$
s-2=\left(d^{2}-2 c\right) /(c-d),
$$

we get that $p$-part of $d$ is less than $p$-part of $c$.

(3) Let $c s=2^{n}$. Suppose $s>2$. Then

$$
2^{n}+(s / 2-1)^{2}=D
$$

is a square of a positive integer $y$. Hence

$$
y-s / 2+1=2^{l}, \quad y+s / 2-1=2^{n-l}, \quad y=2^{l-1}+2^{n-l-1}, \quad s=2^{n-l}-2^{l}+2 .
$$

Since $s$ is a power of 2 , we find $l=n-l$ or $l=1$. If $l=1$, then $s=2^{n-1}$, which implies $c=s=2$, while if $l=n-l$, then $s=2$, a contradiction in both cases.

(4) If $s=2$, then by [3, Proposition 1.17.2] $\widehat{\Delta}$ has type $\mathcal{D}_{2 c}$ (and thus it can be constructed on the points of a projective plane of order $q=2 c=D$ with a polarity $\pi$, whose absolute points form a line $A$ ).

The lemma is proved. 


\section{Automorphisms of $(k+1, r, 1)$-covers}

In this section, we prepare some technical results about automorphisms of $\Delta$, which will be needed for the proof of Theorems 1 and 2 .

The permutation representation of a group $G \leq \operatorname{Aut}(\Delta)$ in its natural action on the vertex set of $\Delta$ gives rise to a matrix representation $G \rightarrow \mathrm{GL}_{v}(\mathbb{C})$. Recall that $\mathbb{C}^{v}$ is the orthogonal direct sum of the eigenspaces $W_{0}, \ldots, W_{3}$ of the adjacency matrix of $\Delta$, where $W_{i}$ corresponds to the eigenvalue $\theta_{i}$. As each $W_{i}$ is a $G$-invariant subspace, it affords a character, say $\chi_{i}$, of $G$. We can calculate values of this character using the theory of association schemes (see [4, Section 3.7]). Namely, let $Q$ be the second eigenmatrix of $\Delta$. (We assume that the first column of $Q$ consists of the multiplicities $m_{i}$ 's.) Then, for an element $g \in G$, one has

$$
\chi_{i}(g)=\frac{1}{v} \sum_{j=0}^{3} Q_{i j} \alpha_{j}(g),
$$

where $\alpha_{j}(g)$ denotes the number of vertices $x$ of $\Delta$ such that $\partial\left(x, x^{g}\right)=j$. Recall that every character value must be an algebraic integer; in particular, if the value is rational, then it is an integer. The second eigenmatrix $Q$ for $\Delta$ was determined in [16].

Lemma 4 [16, Lemma 2.2.2]. If $g \in \operatorname{Aut}(\Delta)$, then

$$
\begin{gathered}
\chi_{1}(g)=\frac{(\sqrt{D}-s / 2+1)}{(c s-s+1)(2 c s \sqrt{D})}\left(\left(c^{2} s^{2}-c s^{2}+s / 2+\sqrt{D}-1\right) \alpha_{0}(g)+(s / 2+\sqrt{D}-1) \times\right. \\
\left.\times(c s-s+1) \alpha_{1}(g)-(c s-s / 2-\sqrt{D}+1) \alpha_{3}(g)\right)-(c s+1) /(2 \sqrt{D}), \\
\chi_{2}(g)=\frac{\alpha_{0}(g)+\alpha_{3}(g)}{c s-s+1}-1 .
\end{gathered}
$$

Lemma 5. If $\alpha_{3}(g)=v$ for an element $g \in \operatorname{Aut}(\Delta)$, then $s>2$.

P r o o f. Suppose $\alpha_{3}(g)=v$ for an element $g \in \operatorname{Aut}(\Delta)$. Then by Lemma 4 we have

$$
\chi_{1}(g)=-\frac{(\sqrt{D}-s / 2+1)(c s+1)}{2 \sqrt{D}} .
$$

Now if $s=2$, then $\chi_{1}(g)=-(c s+1) / 2 \in \mathbb{Z}$, but $c s+1$ is odd, a contradiction. The lemma is proved.

Lemma 6. If $\alpha_{2}(g)=v$ for an element $g \in \operatorname{Aut}(\Delta)$, then $s=2$.

P r o o f. Suppose $\alpha_{2}(g)=v$ for an element $g \in \operatorname{Aut}(\Delta)$. Then by Lemma 4 we have

$$
\chi_{1}(g)=-\frac{c s+1}{2 \sqrt{D}} .
$$

If $s$ is even and $s \geq 4$, then 2 divides $c s+1$, which is an odd number by Lemma 1, a contradiction. Suppose $s$ is odd. Then $c$ is even and $4 D=4(c s+1)+s(s-4)$ divides $(c s+1)^{2}$, hence

$$
(4(c s+1)+s(s-4)) x=(c s+1)^{2}
$$


for an integer $x$. Put $y=(x, c s+1)$. Then $x=a y, c s+1=b y,(s, b y)=1$ and $b>a$. Thus

$$
4 a y+\frac{s(s-4) a}{b}=b y
$$

and $b$ divides $s-4$. Hence $c \geq s>a$ and

$$
4 a+s \frac{(s-4) a}{b y}=b
$$

implying by divides $(s-4) a$. But then $s^{2}+1 \leq c s+1 \leq s(s-4)$, a contradiction. The lemma is proved.

In Lemmas 7-9 it is supposed that there is an element $g \in \operatorname{Aut}(\Delta)$ of prime order $p$ and $\Omega=\operatorname{Fix}(g)$. For a vertex $x \in \Delta$, we put

$$
R_{i}(x)=\left\{y \in[x] \mid \partial\left(y, y^{g}\right)=i\right\},
$$

where $i=0,1,2,3$, and by $F(x)$ we denote the fibre of $\Delta$ containing $x$.

Lemma 7 (cf. [16, Lemma 2.2.3], [12, Theorem $4.10(i)-(i i)]$ ). Suppose $\Omega=\varnothing$. Then

$$
\alpha_{3}(g)=(c s-s+1) t
$$

with $t=\left|\operatorname{Fix}_{\Sigma}(g)\right|$, and $s=2$ or the number $\alpha_{1}(g)+s t / 2-c s-1$ is a multiple of $\sqrt{D}$; moreover, the following statements hold:

(1) if $p=2$, then st is odd, $\alpha_{1}(g)=(c s-s+1)(c s+1-t)$, and if $\alpha_{3}(g)<v$, then $t=s(c-1)$ and the set $\left\{x \in \Delta \mid \partial\left(x, x^{g}\right)=1\right\}$ is the disjoint union of $c s-s+1$ isolated cliques of size $s+1$;

(2) if $p=3$ and $(3, s+1)=1$, then $\alpha_{1}(g)=0$, the number $c s-s+1$ is divisible by 3 ,

$$
\chi_{1}(g)=\frac{-(c s+1)(\sqrt{D}-s / 2+1)+3 l(\sqrt{D}-s / 2)}{2 \sqrt{D}},
$$

where $l=(c s+1-t) / 3$, and $(c s+1)(s / 2-1)-3 l s / 2$ is a multiple of $\sqrt{D}$.

P r o o f. First, note that $(c s-s+1, c s+1)=1$.

If $\partial\left(u, u^{g}\right)=3$ for a vertex $u$, then $F(u)=F\left(u^{g}\right)$ and hence $p$ divides $c s-s+1$ (the size of a fibre). In particular, if $s$ is even, then $p>2$.

By the integrality of $\chi_{2}(g)$, it follows that $\alpha_{3}(g)=(c s-s+1) t$ for a non-negative integer $t$. Further,

$$
\chi_{1}(g)=\frac{\alpha_{1}(g)-(\sqrt{D}-s / 2) t-(c s+1)}{2 \sqrt{D}},
$$

and if $s>2$, then $\alpha_{1}(g)-(\sqrt{D}-s / 2) t-(c s+1)$ is divisible by $2 \sqrt{D}$.

(1) Let $p=2$ and

$$
\Phi=\left\{x \in \Delta \mid \partial\left(x, x^{g}\right)=3\right\}
$$

Note that $\partial\left(x, x^{g}\right) \neq 2$ for any vertex $x$ of $\Delta$ (as otherwise $[x] \cap\left[x^{g}\right] \subseteq \Omega$, which contradicts our assumption), so that

$$
\Delta \backslash \Phi=\left\{x \in \Delta \mid \partial\left(x, x^{g}\right)=1\right\}
$$


and $\alpha_{2}(g)=0$. Since $c s+1$ is odd, $g$ fixes a fibre of $\Delta$ and $s t$ is odd. Also, we have $\alpha_{1}(g)=(c s-s+1)(c s+1-t)$ and

$$
\chi_{1}(g)=\frac{(c s-s+1)(c s+1-t)-(\sqrt{D}-s / 2) t-(c s+1)}{2 \sqrt{D}} \in \mathbb{Z} .
$$

Suppose $x \in \Phi$. Then $[x]=R_{1}(x) \cup R_{3}(x)$ and $\left|R_{3}(x)\right|=t-1$. Note that, for each edge $\{y, z\} \subset[x]$, we have $z \neq y^{g}$ (otherwise $y^{g} \in[x] \cap\left[x^{g}\right]$, which is impossible by assumption).

If $[x]$ contains an edge $\{y, z\} \subset \Delta \backslash \Phi$, then $\left[x^{g}\right]$ contains the edge $\left\{y^{g}, z^{g}\right\} \subset \Delta \backslash \Phi$, and, since $\{y, z\} \subset R_{1}(x)$, we get that $\left\{z, y, y^{g}, z^{g}\right\}$ is a 4-cycle or a clique, a contradiction. Hence each $(s+1)$-clique that contains an edge from $\Delta \backslash \Phi$, is contained in $\Delta \backslash \Phi$ itself. Since $\Delta \backslash \Phi$ is a regular graph of valency $c s-t$ and $\alpha_{1}(g)=|\Delta \backslash \Phi|$, we conclude that $\Delta \backslash \Phi$ is an edge regular graph with $\lambda_{\Delta \backslash \Phi}=s-1$ and the number of its edges equals $(c s+1-s)(c s+1-t)(c s-t) / 2$. It follows that there are exactly $(c s+1-s)(c s+1-t)(c s-t) /((s+1) s)$ cliques of size $s+1$ in $\Delta \backslash \Phi$.

Now suppose that $x \in \Delta \backslash \Phi$. Then $[x]=R_{1}(x) \cup R_{3}(x),\left|R_{3}(x)\right|=t$ and, as it was proved above, $[x] \cap\left[x^{g}\right] \subset \Delta \backslash \Phi$. Note that for each vertex $y \in R_{1}(x) \backslash\left\{x^{g}\right\}$ we have $y^{g} \in\left[x^{g}\right] \cap[y]$ and since $\left\{x, y, y^{g}, x^{g}\right\}$ cannot be a 4-cycle, we get $\left\{y, y^{g}\right\} \subset[x] \cap\left[x^{g}\right]$. This implies $\left|R_{1}(x)\right|=s$. On the other hand, $\left|R_{1}(x)\right|=c s-t$, which, by the preceding equality, implies that $s(c-1)=t$. Hence, there are exactly $c s+1-s$ cliques of size $s+1$ in $\Delta \backslash \Phi$ and $\alpha_{1}(g)=(c s-s+1)(s+1)$, which implies that $\Delta \backslash \Phi$ is the disjoint union of $c s-s+1$ isolated cliques of size $s+1$.

(2) Let $p=3$ and $(3, s+1)=1$. Then $\alpha_{1}(g)=0$ (otherwise there is a (unique) $(s+1)$ clique $L$ that contains a 3 -cycle $\left\{u, u^{g}, u^{g^{2}}\right\}$, yielding $L=L^{g}$, which contradicts the assumption $\Omega=\varnothing$ ). If 3 divides $c s+1$, then $\alpha_{2}(g)=v$ and by Lemma 6 we obtain $s=2$, a contradiction. Assume that there are exactly $3 l$ fibres that are not fixed by $g$. Then $\alpha_{2}(g)=3 l(c s-s+1)$ and $\alpha_{3}(g)=(c s+1-3 l)(c s-s+1)$. Hence

$$
\chi_{1}(g)=\frac{-(c s+1)(\sqrt{D}-s / 2+1)+3 l(\sqrt{D}-s / 2)}{2 \sqrt{D}} \in \mathbb{Z}
$$

and $(c s+1)(s / 2-1)-3 l s / 2$ is a multiple of $\sqrt{D}$. The lemma is proved.

Remark 1. Note that Lemma 7 (1) specifies statements of [12, Theorem $4.10(i),(i i)]$, and Lemma 7 (2) corrects [16, Lemma 2.2.3] (namely, the condition $(3, s+1)=1$ is missing there).

Lemma 8 (see [16, Lemma 2.2.4], [12, Theorem $4.10(i v)-(v i)]$ ). Suppose $\Omega \neq \varnothing$ and $p=2$. Then one of the following statements holds:

(1) $\Omega$ is a fibre of $\Delta$, and either $s=2$, or $c s^{2}-s^{2} / 2+s-\alpha_{1}(g)$ is a multiple of $\sqrt{D}$;

(2) $\Omega$ is an $(s+1)$-clique and $c=s=2$;

(3) $\Omega$ is an $\left(c^{\prime} s^{\prime}+1, c^{\prime} s^{\prime}-s^{\prime}+1,1\right)$-cover, and the parameters $c, s, c^{\prime}$ and $s^{\prime}$ satisfy the following equality:

$$
(c s-s+1)\left(c s-c^{\prime} s^{\prime}\right)=\left(c^{\prime} s^{\prime}+1\right)\left(c^{\prime} s^{\prime}-s^{\prime}+1\right)\left(c s-c^{\prime} s+\frac{c^{\prime}\left(s-s^{\prime}\right)}{\left(s^{\prime}+1\right)}\right)
$$

moreover, $(i) s^{\prime}=2$ and $2 c=\left(2 c^{\prime}\right)^{2}$ if $s=2$, and $(i i) c s-s+1=\left(c^{\prime} s^{\prime}-s^{\prime}+1\right)^{2}$ if $s>s^{\prime}>1$. 
$\mathrm{Pr}$ o o f. Let $p=2$ and $a \in \Omega$. Note that, for each vertex $e \in \Omega \cap \Delta_{3}(a)$, the valency of $e$ in $\Omega$ coincides with that of $a$. Indeed, for each vertex $x \in \Omega_{1}(a)$, there is a unique vertex $x^{\prime} \in[x] \cap[e]$, and $x^{\prime} \in \Omega$. Conversely, $\{x\}=\left[x^{\prime}\right] \cap[a]$.

(1) Suppose that all vertices in $\Omega$ are at pairwise distance 3 in $\Delta$ and $|\Omega|=\omega$. Suppose further that for some vertex $a \in \Omega$ we have $F(a) \backslash \Omega \neq \varnothing$ and let $u \in F(a) \backslash \Omega$. Then $\partial\left(u, u^{g}\right)=3$ and for each vertex $x \in[u]$ we get $x^{\perp} \subset \Delta \backslash \Omega$, hence $\partial\left(x, x^{g}\right) \neq 2$. If $\partial\left(x, x^{g}\right)=3$ for all $x \in[u]$, then by [8, Corollary 6.3] $g=1$, a contradiction. It follows that there is a vertex $x \in[u]$ such that $\partial\left(x, x^{g}\right)=1$ and $s=\left|[x] \cap\left[x^{g}\right]\right|+1$ is odd. Hence $\omega$ is even. Now if, for a vertex $y \in[u], \partial\left(y, y^{g}\right)=3$, then $g$ fixes a vertex in $F(y)$ that has a neighbour in $\Omega$, which contradicts our assumption. It follows that $R_{1}(u)=[u]$. Since $s \geq 3$, we may assume that $[u]$ contains an edge $\{x, y\}$. Then $\left[u^{g}\right]$ contains the edge $\left\{x^{g}, y^{g}\right\}$ and, as $\left[u^{g}\right] \cap[u]=\varnothing$, we get that $\left\{x^{g}, x, y, y^{g}\right\}$ is a 4-cycle, a contradiction.

Hence, $\omega=c s-s+1$, that is $\Omega=F(a)$. Then $\alpha_{3}(g)=0, \alpha_{2}(g)$ is divisible by $2 s, \alpha_{1}(g)=$ $\operatorname{cs} \omega-\alpha_{2}(g)$ is divisible by $2 s$, and

$$
\begin{aligned}
\chi_{1}(g)= & \frac{(\sqrt{D}-s / 2+1)}{(2 c s \sqrt{D})}\left(\left(c^{2} s^{2}-c s^{2}+s / 2+\sqrt{D}-1\right)+(s / 2+\sqrt{D}-1) \alpha_{1}(g)\right)- \\
& (c s+1) /(2 \sqrt{D})=\left((c s-s)(\sqrt{D}-s / 2+1)+\alpha_{1}(g)-c s\right) /(2 \sqrt{D}) .
\end{aligned}
$$

Thus, $s=2$ or $2 \sqrt{D}$ divides $s(c-1)(\sqrt{D}-s / 2+1)+\alpha_{1}(g)-c s$.

(2) Suppose $\Omega$ is an $\omega$-clique. Then $1<\omega \leq s+1$. Suppose further that there is a vertex $x \in \Delta \backslash \Omega$ that has no neighbours in $\Omega$. Clearly, $\partial\left(x, x^{g}\right) \neq 2$, and if $\partial\left(x, x^{g}\right)=1$, then, since $|[a] \backslash \Omega|=c s-s$ is even, we get that $s$ is even and $[x] \cap\left[x^{g}\right]$ contains a vertex from $\Omega$, a contradiction. Hence $\partial\left(x, x^{g}\right)=3$. Furthermore, each vertex of $\Omega$ has exactly $c s-\omega+1$ neighbours in $\Delta \backslash \Omega$, among which there are exactly $c s-s$ vertices that do not belong to the maximal clique of $\Delta$ containing $\Omega$. Hence there are exactly $s-\omega+1+\omega(c s-s)$ vertices in $\Delta \backslash \Omega$ that have a neighbour in $\Omega$. Thus, $\alpha_{1}(g)+\alpha_{2}(g)=s+1-\omega+\omega(c s-s)$ and $\alpha_{3}(g)=\omega(c s-s)$. Then $v=\omega+s+1-\omega+\omega(c s-s)+\omega(c s-s)$, which implies $c=s=2$ and $\omega=3$.

(3) Suppose $\Omega$ contains a pair of vertices $a$ and $b$ such that $\partial(a, b)=2$. Put $[a] \cap[b]=\{c\}$. Then $[a]$ contains a unique vertex $e \in \Delta_{3}(b)$ (which, obviously, belongs to $\Omega$ ) and $\Omega_{1}(b)$ contains a unique vertex $f \in \Delta_{3}(a)$. Further,

$$
\left|\Omega_{1}(a) \cap \Delta_{2}(b) \backslash c^{\perp}\right|=\left|\Omega_{1}(b) \cap \Delta_{2}(a) \backslash c^{\perp}\right| .
$$

Let $X_{1}, \ldots, X_{n}$ denote the fibres that intersect $\Omega$. Then a vertex in $X_{1} \cap \Omega$ has a unique neighbour in each of the fibres $X_{2}, \ldots, X_{n}$, hence $\Omega$ is a regular graph of valency $n-1$ and $|\Omega|=n\left|X_{i} \cap \Omega\right|$. Moreover, $\Omega$ is a $(|\Omega| / n)$-cover of an $n$-clique, in which any two non-adjacent vertices from distinct fibres, say $\Omega \cap X_{i}$ and $\Omega \cap X_{j}$, have exactly one common neighbour. It follows by [8, Lemma 3.1] that $\Omega$ is an $\left(c^{\prime} s^{\prime}+1, c^{\prime} s^{\prime}-s^{\prime}+1,1\right)$-cover, where $c^{\prime} s^{\prime}=n-1$ and, clearly,

$$
s^{\prime}-1=\left|\Omega_{1}(x) \cap \Omega_{1}(y)\right| \equiv s-1 \quad(\bmod p) .
$$

Note that there are exactly $n\left(n-s^{\prime}\right)\left(c s-c^{\prime} s^{\prime}\right)$ edges between $\Omega$ and $\Delta \backslash \Omega$, and there are exactly $c^{\prime} n\left(n-s^{\prime}\right) /\left(s^{\prime}+1\right)$ maximal cliques in $\Omega$. Hence we find that the number of vertices of $\Delta$ that have exactly $s^{\prime}+1$ neighbours in $\Omega$ equals

$$
\tau_{s^{\prime}+1}:=c^{\prime} n\left(n-s^{\prime}\right)\left(s-s^{\prime}\right) /\left(s^{\prime}+1\right),
$$

and the number of vertices of $\Delta$ that have exactly one neighbour in $\Omega$ equals

$$
\tau_{1}:=n\left(n-s^{\prime}\right)\left(c s-c^{\prime} s\right)
$$


Clearly, if there is a vertex $x \in \Delta \backslash \Omega$ that has no neighbour in $\Omega$, then $F(a) \not \subset \Omega$ for all $a \in \Omega$, and, as above, we obtain $\partial\left(x, x^{g}\right) \neq 2$. Put

$$
\Phi=\left\{y \in \Delta \mid \partial\left(y, y^{g}\right)=1, \quad[y] \subset \Delta \backslash \Omega\right\} .
$$

First we prove that $|\Omega|+n=s(c-1)$ in the case $\Phi \neq \varnothing$. Suppose $x \in \Phi$. Since $g$ fixes the subgraph $[x] \cap\left[x^{g}\right]$ and $[x] \subset \Delta \backslash \Omega$, it follows that $s$ is odd. We have $\left|R_{1}(x)\right|=s,\left|R_{3}(x)\right|=n$ (since $\partial\left(w, w^{g}\right)=3$ if and only if $g$ fixes a vertex in $F(w)$ ) and $\left|R_{2}(x)\right|=c s-\left|R_{1}(x)\right|-n$.

Let us compare the sizes of the sets $R_{2}(x)$ and $R_{3}(x)$. As $\Omega$ contains no vertices from $F(x) \cup F\left(x^{g}\right)$, we get that $\Omega$ contains a vertex $b \in \Delta_{2}(x) \cap \Delta_{2}\left(x^{g}\right)$, and, since the number $p_{22}^{1}=s(c-1)(c s-2)$ is even, the number of vertices in $\Delta_{2}(x) \cap \Delta_{2}\left(x^{g}\right) \cap \Omega$ is also even. For the vertex $y \in[b] \cap[x]$ we have $y^{g} \in[b] \cap\left[x^{g}\right]$ and $\partial\left(y, y^{g}\right)=2$ (otherwise $\left\{x, x^{g}, y, y^{g}\right\}$ is a clique and $[b] \cap[x]$ contains $y, y^{g}$, which is impossible). Pick a vertex $w \in[x]$. If $\partial\left(w, w^{g}\right)=2$, then $w, w^{g} \in[a]$ for a vertex $a \in \Omega, x$ has a unique neighbour $u \in F(a), x^{g}$ has a unique neighbour $u^{g} \in F(a)=F(u)$ and $\left\{a, w, x, x^{g}, w^{g}\right\}$ is a 5-cycle. If $\partial\left(w, w^{g}\right)=3$, then $w \in F\left(w^{g}\right)$ and, for each vertex $a \in \Omega$ such that $a \in \Delta_{2}(x) \cap \Delta_{2}\left(x^{g}\right) \cap F(w)$, we get that $\left\{a, u, x, x^{g}, u^{g}\right\}$ is a 5-cycle, where $\{u\}=[a] \cap[x]$. Since for each vertex $w \in R_{3}(x)$ there are exactly $c^{\prime} s^{\prime}-s^{\prime}+1$ vertices in $\Omega \cap F(w)\left(\cap \Delta_{2}(x)\right)$, there are exactly $c^{\prime} s^{\prime}-s^{\prime}+1$ vertices $y$ such that $\{y\}=[x] \cap[a] \subset R_{2}(x)$, where $a \in \Omega \cap F(w)$. Hence,

$$
\left|R_{2}(x)\right|=\left(c^{\prime} s^{\prime}-s^{\prime}+1\right)\left|R_{3}(x)\right|
$$

which implies

$$
s=c s-n\left(c^{\prime} s^{\prime}-s^{\prime}+2\right), \quad \text { and } \quad c s-s=n\left(c^{\prime} s^{\prime}-s^{\prime}+2\right),
$$

that is

$$
|\Omega|+n=s(c-1) .
$$

Now consider the BRG-graph $\widehat{\Delta}$ and note that its corresponding Moore geometry $\mathcal{G}$ has type $(c s+2, c s-s+3, s+1)$ (in notation of [12, p. 314]). Since $\Omega \nsubseteq z^{\perp}$ for any vertex $z \in \widehat{\Delta},\{\hat{b}\} \cup \Omega$ induces a subgeometry of $\mathcal{G}$ (recall, $\hat{b}$ denotes the vertex of $\widehat{\Delta}$ isolated in $B$ ), so by Lemma 7 and [12, Theorem 4.10] we obtain that one of the following three possibilities occurs: $(i) s^{\prime}=1 ;$;ii) $s=s^{\prime}$; (iii) $s>s^{\prime}>1$ and $c s-s+1=\left(c^{\prime} s^{\prime}-s^{\prime}+1\right)^{2}$ (or equivalently, $s(c-1)=s^{\prime}\left(c^{\prime}-1\right)\left(c^{\prime} s^{\prime}-s^{\prime}+2\right)$ ).

Hence $\Phi=\varnothing$ and each vertex in $\Delta \backslash \bigcup_{j} X_{j}$ has exactly one or $s^{\prime}+1$ neighbours in $\Omega$ and, for all vertices $x \in \Delta$ such that $x^{\perp} \subset \Delta \backslash \Omega$ we have $\partial\left(x, x^{g}\right)=3$. Thus,

$$
\alpha_{3}(g)=v-|\Omega|-\tau_{1}-\tau_{s^{\prime}+1},
$$

and, on the other hand,

$$
\alpha_{3}(g)=\left(c^{\prime} s^{\prime}+1\right)\left(c s-s+1-c^{\prime} s^{\prime}+s^{\prime}-1\right)
$$

which together give

$$
(c s-s+1)\left(c s-c^{\prime} s^{\prime}\right)=\left(c^{\prime} s^{\prime}+1\right)\left(c^{\prime} s^{\prime}-s^{\prime}+1\right)\left(c s-c^{\prime} s+\frac{c^{\prime}\left(s-s^{\prime}\right)}{\left(s^{\prime}+1\right)}\right) .
$$

In particular, if $s=2$, then, since $s^{\prime} \leq s$ and

$$
s^{\prime}-1=\left|\Omega_{1}(x) \cap \Omega_{1}(y)\right| \equiv s-1 \quad(\bmod 2),
$$

we get $s^{\prime}=2$ and

$$
c s-s+1=\left(c^{\prime} s^{\prime}+1\right)\left(c^{\prime} s^{\prime}-s^{\prime}+1\right),
$$


so that

$$
2 c-1=\left(2 c^{\prime}+1\right)\left(2 c^{\prime}-1\right) \text { and } 2 c=\left(2 c^{\prime}\right)^{2} .
$$

The lemma is proved.

Remark 2. Note that Lemma 8 specifies statements of [12, Theorem $4.10(i v)-(v i)]$ and of [16, Lemma 2.2.4]. Also, the proof of Lemma 8 fills a gap in the proof of [16, Lemma 2.2.4 (3)], in which the case $\Phi=\varnothing$ was not excluded properly.

Lemma 9 [16, Lemma 2.2.5]. If $\Omega \neq \varnothing$ and $p>2$, then one of the following statements holds:

(1) $\Omega$ is contained in a fibre of $\Delta$;

(2) $\Omega$ is an $\omega$-clique and $\omega \leq s+1$;

(3) $\Omega$ is an $\left(c^{\prime} s^{\prime}+1, c^{\prime} s^{\prime}-s^{\prime}+1,1\right)$-cover, where $c^{\prime} s^{\prime}+1$ is the number of fibres of $\Delta$ intersecting $\Omega$, and $s^{\prime}-1=\left|\Omega_{1}(x) \cap \Omega_{1}(y)\right| \equiv s-1(\bmod p)$.

P r o o f. Let $a \in \Omega$. Then for each vertex $e \in \Omega \cap \Delta_{3}(a)$ we have $\left|\Omega_{1}(a)\right|=\left|\Omega_{1}(e)\right|$.

Clearly, if $\Omega$ consists of vertices that are at pairwise distance 3 in $\Delta$, then the statement (1) is true, while if $\Omega$ is a $\omega$-clique, then $\omega \leq s+1$ and the statement (2) holds.

Now let $\Omega$ contain two vertices $a$ and $b$ such that $\partial(a, b)=2$. Then $[a]$ contains a unique vertex that belongs to $\Delta_{3}(b)$ (and, obviously, to $\Omega$ ) and $\Omega_{1}(b)$ contains a unique vertex that belongs to $\Delta_{3}(a)$. Put $[a] \cap[b]=\{x\}$. Then $\left|\Omega_{1}(a) \cap \Delta_{2}(b) \backslash x^{\perp}\right|=\left|\Omega_{1}(b) \cap \Delta_{2}(a) \backslash x^{\perp}\right|$.

Let $X_{1}, \ldots, X_{n}$ denote the fibres of $\Delta$ that intersect $\Omega$. Then each vertex in $X_{1} \cap \Omega$ has a unique neighbour in each of the fibres $X_{2}, \ldots, X_{n}$, hence $\Omega$ is a regular graph of valency $n-1$ and $|\Omega|=n\left|X_{i} \cap \Omega\right|$. Moreover, $\Omega$ is a $(|\Omega| / n)$-cover of a $n$-clique, in which any two non-adjacent vertices from distinct fibres, say $\Omega \cap X_{i}$ and $\Omega \cap X_{j}$, have exactly one common neighbour. Thus, the remaining claims of (3) follow by [8, Lemma 3.1].

The lemma is proved.

\section{Proofs of Theorems 1 and 2}

In this section, we prove Theorems 1 and 2.

From now on we assume that there is a subgroup $G \leq \operatorname{Aut}(\Delta)$ that induces a 2-homogeneous permutation group $G^{\Sigma}$ on the set $\Sigma$ of fibres of $\Delta$ and we denote by $K$ the kernel of the induced action of $G$, so that $G / K \simeq G^{\Sigma}$. We also put $m:=|\Sigma|=c s+1$.

Note that by [8, Corollary 6.3] $K$ is semiregular, in particular, $\alpha_{3}(g)=v$ for each non-trivial element $g \in K$. It also implies that $K$ acts semiregularly both on the set of arcs of $\Delta$ and on the set of its cliques of size $s+1$. For each subgroup $X \leq K$, we denote by $\Delta^{X}$ the graph on the set of $X$-orbits, whose edges are the (unordered) pairs of $X$-orbits that are joined by an edge of $\Delta$. In particular, if $1<|X|<r$, then by [8, Theorem 6.2 , Corollary 6.3] $\Delta^{X}$ is a non-bipartite $(m,(c s-s+1) /|X|,|X|)$-cover.

First, in Lemma 10, we consider the case, when the group $G^{\Sigma}$ is 2-homogeneous, but not 2 -transitive.

Lemma 10. If the group $G^{\Sigma}$ is not 2-transitive, then $G^{\Sigma} \leq \mathrm{A \Gamma L}_{1}(q),|\Sigma|=q \equiv 3(\bmod 4)$, $\sqrt{D} \notin \mathbb{Z}$, and either $s=2, c=(q-1) / 2$ and $\widehat{\Delta}$ is a graph of type $\mathcal{D}_{q-1}$, or $s$ is odd. 
P r o o f. Suppose that $G$ induces 2-homogeneous, but not 2-transitive permutation group $G^{\Sigma}$ on $\Sigma$. As $m \geq 5$ is odd, it follows by [11, Theorem 1] that $G / K \simeq G^{\Sigma} \leq \mathrm{A} L_{1}(q)$ and $|\Sigma|=q \equiv 3(\bmod 4)$. If $\sqrt{D} \in \mathbb{Z}$, then $c s=D-(s / 2-1)^{2}$ is an even difference of squares, which is impossible in this case. Hence $\sqrt{D} \notin \mathbb{Z}$ and either $s=2$ or $c=(q-1) / 2$ is odd, or $s$ is odd. Finally, if $s=2$, then by Lemma 3 we obtain that $\widehat{\Delta}$ is a graph of type $\mathcal{D}_{q-1}$. The lemma is proved.

Further in Lemmas 11-14, we assume that the group $G^{\Sigma}$ is 2-transitive; in this case by a Burnside's theorem, the group $G^{\Sigma}$ is either almost simple, or affine, and we consider the corresponding cases in course, basing our argument on the classification of finite 2-transitive permutation groups (e.g. see [9, Theorem 2.9]).

Lemma 11. Suppose the group $G^{\Sigma}$ is almost simple. Then either $c=2^{n-1}, s=2, \widehat{\Delta}$ is a graph of type $\mathcal{D}_{2^{n}}, \operatorname{Soc}(G) \simeq \mathrm{L}_{2}\left(2^{n}\right)$ and $\Delta$ is a Mathon graph, or the group $G$ acts intransitively on the vertices of $\Delta$.

P r o o f. Suppose that $G$ induces an almost simple permutation group $G^{\Sigma}$ on $\Sigma$. Then the socle $H$ of $G^{\Sigma}$ is a non-abelian simple group. In view of Lemma 1, we may assume that $s=2$ (and the size of a fibre is $m-2$ ) or $|\Sigma| \geq 25$. Fix $F \in \Sigma$ and $a \in F$.

If $H=\operatorname{Sp}_{2 n}(2)$, then the number $m \in\left\{2^{n-1}\left(2^{n} \pm 1\right)\right\}$ is even, a contradiction.

In the case $H={ }^{2} \mathrm{G}_{2}(q)$ we have $q=3^{2 e+1}$ and $m=q^{3}+1$ is even, a contradiction.

In the case $H=\mathrm{U}_{3}(q)$ we have $m=q^{3}+1$, hence $q=2^{e}$. By Lemma 3 we have $s=2$ and $\widehat{\Delta}$ is a graph of type $\mathcal{D}_{2^{3 e}}$. Then by Lemma 5 we have $K=1$ and, since $G_{\{F\}}$ contains no subgroup of index $q^{3}-1, G$ cannot act transitively on the vertices of $\Delta$.

In the case $H={ }^{2} \mathrm{~B}_{2}(q)$ we have $q=2^{2 e+1}$ and $m=q^{2}+1$. By Lemma 3 we get $s=2$ and $\widehat{\Delta}$ has type $\mathcal{D}_{2^{2(2 e+1)}}$. By Lemma 5 it follows that $K=1$ and, since $G_{\{F\}}$ contains no subgroup of index $q^{2}-1, G$ cannot act transitively on the vertices of $\Delta$.

If $H$ is a Mathieu group $\mathrm{M}_{m}$, then (since $m$ is odd) $m \in\{11,23\}$ and, by Lemma 1 we have $s=2$.

If the pair $(H, m)$ is one of $\left(\mathrm{L}_{2}(11), 11\right),\left(\mathrm{M}_{11}, 12\right),\left(\mathrm{Alt}_{7}, 15\right),\left(\mathrm{L}_{2}(8), 28\right)$, (HiS, 176), or $\left(\mathrm{Co}_{3}, 276\right)$, then (since $m$ is odd) $m=11, H=\mathrm{L}_{2}(11)$ or $m=15, H=\mathrm{Alt}_{7}$ and, by Lemma 1 we have $s=2$.

If $m=23$, then by [8, Theorem 5.4] $(-1)^{c-1}(2 c-1)=21 \equiv z^{2}(\bmod 11)$ for some $z \in \mathbb{Z}$ and by the Euler's criterion $21^{5} \equiv 1(\bmod 11)$, a contradiction.

Similarly, for $m=15$ by [8, Theorem 5.4] we get $(-1)^{c-1}(2 c-1)=13 \equiv z^{2}(\bmod 7)$ for some $z \in \mathbb{Z}$ and by the Euler's criterion $13^{3} \equiv 1(\bmod 7)$, a contradiction.

If $m=11$, then by Lemma 5 we have $G^{\Sigma} \simeq G$, and either $H=\mathrm{L}_{2}(11)$ and $H_{\{F\}} \simeq \mathrm{Alt}_{5}$, or $H=\mathrm{M}_{11}$ and $H_{\{F\}} \simeq \operatorname{Alt}_{6}: \mathrm{Z}_{2}$. But in both cases $G$ contains no subgroup of index 99 , hence $G$ cannot act transitively on the vertices of $\Delta$.

It remains to consider "alternating" and "linear" cases.

1. Let $H=\operatorname{Alt}_{m}$. Then $H$ contains an involution $g$ that is a product of two independent transpositions and the number of the fibres that are fixed by $g$ equals $m-4$.

Note that for $m=5$ we have $c=s=2$ and by Lemma 5 we have $\mathrm{L}_{2}(4) \simeq \operatorname{Alt}_{5} \unlhd G^{\Sigma} \simeq G$, and, moreover, if $G$ is vertex-transitive, then it has a single orbit on arcs of $\Delta$ as well. So let $m \geq 7$.

1.1. First, suppose $K=1$. Then $G \simeq G^{\Sigma}$ and we may identify $H$ with the socle of $G$.

Put $\Omega=\operatorname{Fix}(g)$. If $\Omega=\varnothing$, then by Lemma 7 we obtain $m-4=s(c-1)$, that is $s=3, m \geq 13$. In this case, $H$ contains an involution $g^{\prime}$ that is a product of four independent transpositions and the number of the fibres that are fixed by $g^{\prime}$ equals $m-8$, and by Lemma 7 we have $\Omega^{\prime}=\operatorname{Fix}\left(g^{\prime}\right) \neq \varnothing$ (otherwise $m-8=3(c-1)$, which is impossible). Hence $\Omega^{\prime}$ is distance-regular and its parameters 
satisfy the equality given in Lemma $8(3)$, which, in view of Lemma 1, contradicts the restriction $m \geq 13$.

Hence by Lemma 8 we obtain that $\Omega$ is an $\left(c^{\prime} s^{\prime}+1, c^{\prime} s^{\prime}-s^{\prime}+1,1\right)$-cover,

$$
4(m-s)=(m-4)\left(m-s^{\prime}\right)\left(m-1-c^{\prime} s+\frac{c^{\prime}\left(s-s^{\prime}\right)}{\left(s^{\prime}+1\right)}\right)
$$

and $m=7$, that is $c^{\prime} s^{\prime}=2$ and $\Omega$ is a 6 -cycle. But $s-1 \equiv s^{\prime}-1(\bmod 2)$ and hence $\Delta$ has intersection array $\{6,5,1 ; 1,1,6\}$, which contradicts the assumption $s>1$.

1.2. Now let $K>1$. If $|K|$ is odd or coincides with the size of a fibre (so that $G_{\{F\}}=K: G_{a}$ ), then there are involutions $g \in G \backslash K$ with $\left|\operatorname{Fix}_{\Sigma}(g)\right|=m-4$ or $m-8$, and we proceed as in the subcase 1.1.

Suppose that $1<|K|<c s-s+1$ and $G$ acts transitively on vertices of $\Delta$. Then

$$
\operatorname{Alt}_{m-1} \leq\left(G_{\{F\}}\right)^{\Sigma}\left(\simeq G_{\{F\}} / K\right) \leq \operatorname{Sym}_{m-1},
$$

and the graph $\Delta^{K}$ admits a vertex-transitive action of $G / K$, and the size of a fibre in $\Delta_{1}=\Delta^{K}$ is $r^{\prime}=(c s-s+1) /|K|$. If $r^{\prime}=2$, then $G / K$ is a distance-transitive group of automorphisms of $\Delta_{1}$ with $\operatorname{Alt}_{m-1} \leq G_{a} K / K \leq\left(\operatorname{Aut}\left(\Delta_{1}\right)\right)_{x}$ for some vertex $x \in \Delta_{1}$, which implies that $\Delta_{1}$ is bipartite, a contradiction. Hence $r^{\prime} \geq 3$. But the degree of a minimal permutation representation of Alt $_{m-1}$ is $m-1$ unless $m \leq 5$, so we obtain either $m=5$ and $s=1$, or $m \geq 7$ and

$$
\operatorname{Alt}_{m-1} \leq G_{a} K / K \leq\left(\operatorname{Aut}\left(\Delta_{1}\right)\right)_{x}
$$

for some vertex $x \in \Delta_{1}$ (and hence $r^{\prime}=\left|G_{\{F\}} / K: G_{a} K / K\right| \leq 2$ ), a contradiction in both cases.

2. Next we assume $H=\mathrm{L}_{d}(q)$. Then $\Sigma$ can be regarded as the set of 1-dimensional subspaces of $V=\mathbb{F}_{q}{ }^{d}$. Note that, since

$$
m=\frac{\left(q^{d}-1\right)}{(q-1)}
$$

must be odd, $q$ is even or $d$ is odd.

Let $d=2$. Then $q=2^{n}, m=q+1$ and by Lemma 3 we have $s=2$, which by Lemma 5 implies $\mathrm{L}_{2}(q) \unlhd G \leq \mathrm{P}^{2} \mathrm{~L}_{2}(q)$. Note if the group $G$ is vertex-transitive, then it has a single orbit on arcs of $\Delta$, and moreover, its socle is also arc-transitive (otherwise $\Delta$ would be bipartite or disconnected, which is impossible), and hence $\Delta$ is a Mathon graph (see [3, Proposition 12.5.3]).

Suppose further that $d \geq 3$ and fix a basis $e_{1}, e_{2}, \ldots, e_{d}$ of $V$.

2.1. Assume first that $K=1$. In the argument below, we identify $H$ with the socle of $G$ and consider various involutions $g \in H$ and subgraphs $\Omega=\operatorname{Fix}(g)$ of their fixed points.

2.1.1. Suppose $q$ is odd. Then $d$ is odd and there is an involution $g \in H$ such that its preimage in $\mathrm{SL}(V)$ fixes $e_{d}$ and, for all $i \in\{1,2, \ldots, d-1\}$, it maps $e_{i}$ to $-e_{i}$, so that

$$
\left|\operatorname{Fix}_{\Sigma}(g)\right|=\frac{\left(q^{d-1}-1\right)}{(q-1)}+1 .
$$

If $\Omega=\varnothing$, then $s$ is odd and by Lemma 7 we have

$$
\frac{\left(q^{d-1}-1\right)}{(q-1)}+1=s(c-1) .
$$

But then

$$
s=s c+1-s(c-1)-1=\frac{\left(q^{d}-1\right)}{(q-1)}-\frac{\left(q^{d-1}-1\right)}{(q-1)}-2=q^{d-1}-2
$$


and

$$
\frac{\left(q^{d-1}-1\right)}{(q-1)}+1=\left(q^{d-1}-2\right)(c-1),
$$

which contradicts the condition $c \geq s>2$.

Hence by Lemma 8 we have that $\Omega$ is an $\left(\left(q^{d-1}-1\right) /(q-1)+1,\left(q^{d-1}-1\right) /(q-1)-s^{\prime}, 1\right)$-cover and

$$
\left(q^{d-1}-1\right)\left(\frac{\left(q^{d}-1\right)}{(q-1)}-s\right)=\left(\frac{\left(q^{d-1}-1\right)}{(q-1)}+1\right)\left(\frac{\left(q^{d-1}-1\right)}{(q-1)}-s^{\prime}+1\right)\left(c s-c^{\prime} s+\frac{c^{\prime}\left(s-s^{\prime}\right)}{\left(s^{\prime}+1\right)}\right) .
$$

Hence $\left(q^{d-1}-1\right) /(q-1)+1$ divides $(q-1)(s+q-1), d=3$ and $s=(q+11) / 3$. But $(q+11,3 q(q+1))$ divides 330 , which implies that the corresponding equation has no solution in natural numbers, a contradiction.

2.1.2. Now let $q$ be even.

2.1.2 (a). If $d=2 f+1$, then we assume that a preimage of $g$ in $\operatorname{SL}(V)$ fixes $e_{d}$ and, for $1 \leq i \leq d-1$, interchanges $e_{i}$ with $e_{d-1-i}$. Then

$$
\left|\operatorname{Fix}_{\Sigma}(g)\right|=\frac{\left(q^{f+1}-1\right)}{(q-1)} .
$$

If $\Omega=\varnothing$, then $s$ is odd and by Lemma 7 we have

$$
\frac{\left(q^{f+1}-1\right)}{(q-1)}=s(c-1)
$$

that is

$$
s=q^{d-1}+\ldots+q^{f+1}-1
$$

and

$$
\frac{\left(q^{f+1}-1\right)}{(q-1)}=\left(q^{d-1}+\ldots+q^{f+1}-1\right)(c-1),
$$

which contradicts the condition $c>2$.

Hence, by Lemma 8 we have that $\Omega$ is an $\left(\left(q^{f+1}-1\right) /(q-1),\left(q^{f+1}-1\right) /(q-1)-s^{\prime}-1,1\right)$-cover and

$$
\left(\frac{\left(q^{2 f+1}-1\right)}{(q-1)}-s\right) q^{f+1} \frac{\left(q^{f}-1\right)}{(q-1)}=\frac{\left(q^{f+1}-1\right)}{(q-1)} \cdot\left(\frac{\left(q^{f+1}-1\right)}{(q-1)}-s^{\prime}\right)\left(c s-c^{\prime} s+\frac{c^{\prime}\left(s-s^{\prime}\right)}{\left(s^{\prime}+1\right)}\right) .
$$

Hence

$$
s=q^{f-1}+\ldots+q+1, \quad s+q^{f}=s q+1, \quad c=q\left(q^{f}+1\right)
$$

and

$$
q^{2 f+1} \frac{\left(q^{f}-1\right)}{(q-1)}=\left(\frac{\left(q^{f+1}-1\right)}{(q-1)}-s^{\prime}\right)\left(c s-c^{\prime} s+\frac{c^{\prime}\left(s-s^{\prime}\right)}{\left(s^{\prime}+1\right)}\right)
$$

and, since $q^{f}>s \geq s^{\prime}$, we get $f=s=1$, a contradiction.

2.1.2 (b). For $d=2 f$, we assume that a preimage of $g$ in $\operatorname{SL}(V)$ fixes both $e_{d-1}$ and $e_{d}$, and interchanges $e_{i}$ with $e_{d-2-i}$, so that

$$
\left|\operatorname{Fix}_{\Sigma}(g)\right|=\frac{\left(q^{f+1}-1\right)}{(q-1)}
$$


If $\Omega=\varnothing$, then $s$ is odd and by Lemma 7 we have

$$
\frac{\left(q^{f+1}-1\right)}{(q-1)}=s(c-1)
$$

that is

$$
s=q^{d-1}+\ldots+q^{f+1}-1
$$

and

$$
\frac{\left(q^{f+1}-1\right)}{(q-1)}=\left(q^{d-1}+\ldots+q^{f+1}-1\right)(c-1),
$$

which contradicts the condition $c>2$.

Hence, by Lemma $8, \Omega$ is an $\left(\left(q^{f+1}-1\right) /(q-1),\left(q^{f+1}-1\right) /(q-1)-s^{\prime}, 1\right)$-cover and

$$
\left(\frac{\left(q^{2 f}-1\right)}{(q-1)}-s\right) q^{f+1} \frac{\left(q^{f-1}-1\right)}{(q-1)}=\frac{\left(q^{f+1}-1\right)}{(q-1)} \cdot\left(\frac{\left(q^{f+1}-1\right)}{(q-1)}-s^{\prime}\right)\left(c s-c^{\prime} s+\frac{c^{\prime}\left(s-s^{\prime}\right)}{\left(s^{\prime}+1\right)}\right) .
$$

If

$$
\left(\frac{\left(q^{f+1}-1\right)}{(q-1)}, \frac{\left(q^{f-1}-1\right)}{(q-1)}\right)=1
$$

then $f$ is even, $q^{f}+\ldots+q+1$ divides $s q^{2}+q+1, s q^{2}=x\left(q^{f}+\ldots+q+1\right)-q-1, q^{2}$ divides $x-1$ and

$$
s \geq\left(q^{2}+1\right)\left(q^{f-2}+\ldots+q+1\right)+q+1 .
$$

But $s \leq c-1$ and $c s=q^{2 f-1}+\ldots+q^{2}+q$, a contradiction.

Hence, $f$ is odd and

$$
\left(\frac{\left(q^{f+1}-1\right)}{(q-1)}, \frac{\left(q^{f-1}-1\right)}{(q-1)}\right)=q+1 .
$$

It follows that $\left(q^{f+1}-1\right) /\left(q^{2}-1\right)$ divides $s q^{2}+q+1, s q^{2}=x\left(q^{f}+\ldots+q+1\right)-q-1, q$ divides $x-1, q^{2}$ divides $x-(q+1)$ and $x=z q^{2}+q+1$ for a positive integer $z$. But

$$
\frac{\left(q^{2 f}-1\right)}{(q-1)}>s^{2}>\frac{z^{2}\left(q^{f+1}-1\right)^{2}}{\left(q^{2}-1\right)^{2}}>q^{2 f}
$$

again a contradiction.

2.2. Now let $K>1$. If $|K|$ is odd or coincides with the size of a fibre, then there are involutions $g \in G \backslash K$ with $\left|\operatorname{Fix}_{\Sigma}(g)\right|$ as in the subcase 2.1, and by a similar argument we come to a contradiction.

Suppose that $1<|K|<c s-s+1, s$ is odd and $G$ acts transitively on vertices of $\Delta$.

Let $\widetilde{H}$ denote the full preimage of $H\left(=\operatorname{Soc}\left(G^{\Sigma}\right)\right)$ in $G$ and put $t=\left|\widetilde{H}_{\{F\}}: \widetilde{H}_{a}\right|$. We have

$$
G^{\Sigma} \leq \mathrm{P} \mathrm{L}_{d}(q), \quad \widetilde{H}_{\{F\}} / K \simeq\left(\widetilde{H}_{\{F\}}\right)^{\Sigma} \simeq \mathrm{E}_{q^{d-1}} \cdot \mathrm{SL}_{d-1}(q) \cdot \mathrm{Z}_{(q-1) /(d, q-1)}
$$

(e.g. see [18]), and $\left(\widetilde{H}_{a}\right)^{[a]}$ is permutation isomorphic to $\left(\widetilde{H}_{a} K / K\right)^{\Sigma \backslash\{F\}}$. So

$$
\frac{(c s-s+1)}{t}\left|G_{a}: \widetilde{H}_{a}\right|=|G: \widetilde{H}|=\left|G^{\Sigma}: H^{\Sigma}\right|
$$

divides $(q-1) e$ (where $q=p^{e}$ for a prime $p$ ), and $\left|\widetilde{H}_{\{F\}}: \widetilde{H}_{a} K\right|=t /|K|$. Hence $\widetilde{H}_{a} \simeq \widetilde{H}_{a} K / K$ is isomorphic to a subgroup of $\mathrm{E}_{q^{d-1}} \cdot \mathrm{SL}_{d-1}(q) \cdot \mathrm{Z}_{(q-1) /(d, q-1)}$ with index $t /|K|$ dividing

$$
\left(\frac{\left(q^{d}-1\right)}{(q-1)}-s\right) /|K| \text {. }
$$


Since $\widetilde{H}_{\{F\}} / K$ contains a normal elementary abelian group $R$ of size $q^{d-1}$ that corresponds to a subgroup of $\mathrm{SL}(V)$, generated by transvections $g$ with $g\left(e_{1}\right)=e_{1}$ and $g(u)-u \in\left\langle e_{1}\right\rangle$ for all $u \in V$, we may assume $R \cap \widetilde{H}_{a} K / K \neq 1$ (otherwise $q^{d-1}$ divides

$$
t /|K|=\frac{\left(q^{d}-1\right)}{(q-1)}-s=q^{d-1}+\frac{\left(q^{d-1}-1\right)}{(q-1)}-s,
$$

a contradiction).

Let $q$ and $d$ be odd. Then there is an element $h \in \widetilde{H}_{a}$ of order $p$ such that

$$
\left|\operatorname{Fix}_{\Sigma}(h)\right|=\frac{\left(q^{d-1}-1\right)}{(q-1)},
$$

and, since the valency of $\Omega=\operatorname{Fix}(h)$ is odd, by Lemma $9 \Omega$ is a clique of size

$$
\frac{\left(q^{d-1}-1\right)}{(q-1)} \leq s+1
$$

a contradiction.

Let $q$ be even. Then there is an involution $h \in \widetilde{H}_{a}$ such that

$$
\left|\operatorname{Fix}_{\Sigma}(h)\right|=\frac{\left(q^{d-1}-1\right)}{(q-1)},
$$

and, by Lemma $8 \Omega=\operatorname{Fix}(h)$ is an $\left(\left(q^{d-1}-1\right) /(q-1),\left(q^{d-1}-1\right) /(q-1)-s^{\prime}, 1\right)$-cover. Finally, it is easy to check that the equality given by Lemma $8(3)$ is not satisfied in this case, a contradiction.

The lemma is proved.

Lemma 12. If the group $G^{\Sigma}$ is affine and the group $G$ acts transitively on arcs of $\Delta$, then $s+1$ divides $c\left(p^{e}, c-1\right), s>2,|K|=c s-s+1$ and $|K|$ is divisible by $1+l p$, where $c s+1=p^{e}$, $p$ is a prime and $l$ is a positive integer.

P r o o f. Let $c s+1=p^{e}$ for a prime $p$, and denote by $T$ the full preimage of $\operatorname{Soc}\left(G^{\Sigma}\right)$ in $G$. Since $K$ acts semiregularly on each fibre, $(|K|, c s+1)=1$ and hence each element $g \in T$ of order $p$ has no fixed points. Besides, $K$ has a complement $T_{0}$ in $T$ that is an elementary abelian group of order $p^{e}$, and $K=O^{p}(T)$. Hence, by [1,37.7], $N_{T}\left(T_{0}\right)=C_{T}\left(T_{0}\right)$.

Suppose that $G$ acts transitively on arcs of $\Delta$. Pick $F \in \Sigma$. Then for each vertex $a \in F$ the group $G_{a}$ acts transitively on $[a]$. We have $T_{\{F\}}=K$ and $\left|T: T_{a}\right|=(c s+1)|K|$. Hence $T$ acts transitively on the vertices of $\Delta$ if and only if $K$ acts transitively on $F$.

Suppose that $K$ acts intransitively on $F$. Since $T$-orbits comprise an imprimitivity system of $G$, each $T$-orbit is a coclique (otherwise, a $T$-orbit containing an edge induces a subgraph of valency cs in $\Delta$, which is impossible by assumption). Hence $\alpha_{2}(g)=v$ for each element $g \in T$ of order $p$, and by Lemmas 6 and 5 we have $s=2$ and $K=1$.

Then $|F|=2 c-1, T$ is a subgroup of order $p^{e}$, normal in $G$, and each $T$-orbit contains a unique vertex from every fibre of $\Delta$. Note that $T$ acts semiregularly on the set of 3-cliques of $\Delta$. Hence the number of 3 -cliques of $\Delta$ is divisible by $2 c+1$, which implies 3 divides $c(c-2)$, and $p>3$.

Further, there are exactly $t(t<c s-s+1) T$-orbits that intersect $[a]$, so that $c s=t j$ for some positive integer $j$. Let us show that $t=c$. Indeed, $G_{a}$ acts transitively on the set of non-trivial elements of $T$ and the group $T G_{a}$ induces 2-transitive permutation group of degree $p^{e}$ on $a^{T}$. Now, since each vertex of $[a]$ is adjacent to exactly $j-1$ vertices from $a^{T} \backslash\{a\}$ and there are exactly 
$c s(j-1)$ edges between $[a]$ and $a^{T} \backslash\{a\}$, each vertex from $a^{T} \backslash\{a\}$ is adjacent "on the average" to $j-1$ vertices from $[a]$. Hence $j-1=1$ and $c s=2 t$, that is $t=c$.

Denote by $a_{1}, \ldots, a_{c}$ the vertices of $F$ that have a neighbour in $a^{T} \backslash\{a\}$. Then the set $\sigma=\left\{\left[a_{i}\right] \cap a^{T}\right\}_{i=1}^{c}$ comprises an imprimitivity system of $G_{a}$ on $a^{T} \backslash\{a\}$, and the set $\pi=\left\{[a] \cap a_{i}^{T}\right\}_{i=1}^{c}$ comprises an imprimitivity system of $G_{a}$ on $[a]$.

For a block $\{x, y\} \in \pi$, there is a 2-element $h \in G_{a}$ that interchanges the vertices $x$ and $y$, so $x=y^{h}$ and $h^{2} \in G_{a, x, y}$, and hence, $G_{a}$ contains an involution $z$ that fixes $\{x, y\}$.

Suppose $c>2$. Put $\Omega=\operatorname{Fix}(z)$. Then $F \backslash\left\{a, a_{1}, \ldots, a_{c}\right\} \neq \varnothing$ and by Lemma 8 we have either $\Omega=F$, or $\Omega$ is an $\left(2 c^{\prime}+1,2 c^{\prime}-1,1\right)$-cover and $2 c=\left(2 c^{\prime}\right)^{2}$. But in the second case by Lemma 4 we obtain $\chi_{1}(g)=-(2 c+1) /\left(4 c^{\prime}\right) \in \mathbb{Z}$ for an element $g \in T$ of order $p$, a contradiction. Hence $\langle z\rangle \leq G_{F} \unlhd G_{\{F\}}$ and $G_{a, x}\left(=G_{a, x, y}\right)$ is a $2^{\prime}$-group, that is $h^{2}=1$ and we may assume that $h=z$. It implies that $h$ also fixes the block $\left\{x^{\prime}, y^{\prime}\right\} \in \pi$ with $\left\{x^{\prime}\right\}=[a] \cap[x]$ and $\left\{y^{\prime}\right\}=[a] \cap[y]$, and $(|G|)_{2}$ divides $2 c$.

Thus, for each edge $\left\{x, x^{\prime}\right\} \subset[a]$, there is a (unique) edge $\left\{y, y^{\prime}\right\} \subset[a]$ such that $\{x, y\} \in \pi$ and $\left\{x^{\prime}, y^{\prime}\right\} \in \pi$. On the other hand, for a block $\{x, y\} \in \pi$, there is an element $h \in G_{a}$ such that $\partial\left(x, x^{h}\right)=1$, and, since $[a]$ is the disjoint union of edges, $h^{2} \in G_{a, x, y}$. Without loss of generality, we may assume that $h$ is a 2-element. But $G_{a, x}$ is a $2^{\prime}$-group, that is $h$ is an involution of $G_{a}$ such that $\Omega=\operatorname{Fix}(h) \neq F$ (since $h$ interchanges distinct orbits $x^{T}$ and $\left.\left(x^{\prime}\right)^{T}\right)$. By Lemma 8 it follows that $\Omega$ is an $\left(2 c^{\prime}+1,2 c^{\prime}-1,1\right)$-cover and $2 c=\left(2 c^{\prime}\right)^{2}$, which together with Lemma 4 imply $\chi_{1}(g)=-(2 c+1) /\left(4 c^{\prime}\right) \in \mathbb{Z}$ for an element $g \in T$ of order $p$, a contradiction.

Hence $c=s=2$ and again by Lemma 4 we obtain $\chi_{1}(g)=-(2 c+1) /(2 \sqrt{2 c}) \in \mathbb{Z}$ for an element $g \in T$ of order 5 , a contradiction.

Now suppose that $K$ acts transitively on $F$. Then $G_{\{F\}}=K: G_{a}$ and, by Lemma 5 we have $s>2$. Since $K$ acts semiregularly on the set of cliques of size $s+1$ of $\Delta$, the number of cliques of size $s+1$ of $\Delta$ is divisible by $c s-s+1$ and hence $s+1$ divides $c\left(p^{e}, c-1\right)$.

Further, in view of Lemmas 6 and 2, for each element $g \in T$ of order $p$ there is a $\langle g\rangle$-orbit that is a cycle or a clique. Besides, $\left|\operatorname{Syl}_{p}(T)\right|$ divides $|K|$. If $\left|\operatorname{Syl}_{p}(T)\right|=1$, then $T_{0}=O_{p}(T)$ and, hence, $T_{0} \unlhd G$. But in this case, each $T_{0}$-orbit contains an edge and hence induces a subgraph of valency $c s$ of $\Delta$, a contradiction. Therefore, $|K|$ is divisible by $1+l p$ for some positive integer $l$. The lemma is proved.

Further for a finite group $X$ we denote by $X^{(\infty)}$ the last term of the commutator series of $X$.

Lemma 13. Suppose that the group $G^{\Sigma}$ is affine. Then the group $G$ acts intransitively on arcs of $\Delta$ or $G^{\Sigma} \leq \operatorname{A\Gamma L}_{1}(q)$.

P r o o f. Note that $G^{\Sigma}$ can be identified with a subgroup of $\mathrm{A} \mathrm{L}_{d}(q)$, where $q$ is a power of an odd prime $p$. Thus, the socle $T$ of $G^{\Sigma}$ is regarded as the additive group of a linear space $V$ with dimension $d$ over $\mathbb{F}_{q}, c s=q^{d}-1$, and the stabiliser $G_{0}$ of the zero vector in $G^{\Sigma}$ acts transitively on the set of non-zero vectors of $V$. Fix a basis $e_{1}, \ldots, e_{d}$ of $V$.

First, the case $G_{0}^{(\infty)}=G_{2}(q)$, as well as the cases $G_{0} \in\left\{\operatorname{Alt}_{6}, \operatorname{Alt}_{7}, \mathrm{U}_{3}(3)\right\}$ are immediately ruled out, since $m=|\Sigma|$ must be odd.

Suppose further that $G$ acts transitively on arcs of $\Delta$. Take $F \in \Sigma$. Then by Lemma 5 we have $|K|=c s-s+1, s>2$ and $G_{0} \simeq G_{\{F\}} / K \simeq G_{a}$ for each vertex $a \in F$, thus we may identify the groups $G_{a}{ }^{[a]}$ and $G_{0} V \backslash\{0\}$ in what follows.

Since $K$ acts regularly on each fibre, $(|K|, c s+1)=1$. Hence the full preimage $T$ of $\operatorname{Soc}\left(G^{\Sigma}\right)$ in $G$ contains an element $y$ of order $p$ that has no fixed points, and by Lemma 2 we obtain that each $\langle y\rangle$-orbit containing an edge is a cycle or a clique, while by Lemma 6 we have $\alpha_{2}(y)<v$. 
Note if some $\langle y\rangle$-orbit is a clique, then $y$ fixes an $(s+1)$-clique containing it, implying $p$ divides $(c-1, s+1)$.

1. Suppose $G_{0}{ }^{(\infty)}=\operatorname{SL}_{d}(q)$ or $\operatorname{Sp}_{d}(q)$, where $d \geq 2$, and take an involution $g \in G_{0}{ }^{(\infty)}$ that maps $e_{i}$ to $-e_{i}$ for $1 \leq i \leq 2$ and fixes $e_{i}$ when $i \geq 3$ (in case $G_{0}{ }^{(\infty)}=\operatorname{Sp}_{d}(q)$ we assume that $\left(e_{1}, e_{2}\right)$ is a hyperbolic pair). Then the number of fibres that are fixed by $g$ equals $q^{d-2}$. Put $\Omega=\operatorname{Fix}(g)$.

By Lemma 8 we obtain that $\Omega$ is an $\left(c^{\prime} s^{\prime}+1, c^{\prime} s^{\prime}-s^{\prime}-1,1\right)$-cover, $c^{\prime} s^{\prime}=q^{d-2}-1$ and

$$
\left(q^{2}-1\right)\left(q^{d}-s\right)=\left(q^{d-2}-s^{\prime}\right)\left(c s-c^{\prime} s+\frac{c^{\prime}\left(s-s^{\prime}\right)}{\left(s^{\prime}+1\right)}\right) .
$$

Thus, $q^{d-2}-s^{\prime}$ divides $\left(q^{2}-1\right)\left(q^{2} s^{\prime}-s\right)$ and $3 \neq d \leq 8$.

For $5 \leq d \leq 8$, there is an involution $g^{\prime} \in G_{0}(\infty)$ that maps $e_{i}$ to $-e_{i}$ if $1 \leq i \leq 4$ and fixes $e_{i}$ if $i \geq 5$ (in case $G_{0}{ }^{(\infty)}=\operatorname{Sp}_{d}(q)$ we assume that $\left(e_{1}, e_{2}\right)$ and $\left(e_{3}, e_{4}\right)$ are hyperbolic pairs). The number of fibres that are fixed by $g^{\prime}$ equals $q^{d-4}$, and, again by Lemma $8, \Omega^{\prime}=\operatorname{Fix}\left(g^{\prime}\right)$ is an $\left(c^{\prime \prime} s^{\prime \prime}+1, c^{\prime \prime} s^{\prime \prime}-s^{\prime}-1,1\right)$-cover, $c^{\prime \prime} s^{\prime \prime}=q^{d-4}-1$ and

$$
\left(q^{4}-1\right)\left(q^{d}-s\right)=\left(q^{d-4}-s^{\prime \prime}\right)\left(c s-c^{\prime \prime} s+\frac{c^{\prime \prime}\left(s-s^{\prime \prime}\right)}{\left(s^{\prime \prime}+1\right)}\right),
$$

which contradicts the assumption $5 \leq d \leq 8$.

If $d=4$, then there is an involution $g^{\prime} \in G_{0}{ }^{(\infty)}$ that fixes both $e_{3}$ and $e_{4}$, and, for $1 \leq i \leq 2$, maps $e_{i}$ to $-e_{i}$, so that there are exactly $q^{2}$ fibres that intersect $\Omega^{\prime}=\operatorname{Fix}\left(g^{\prime}\right)$, and, by Lemma 8 , $\Omega^{\prime}$ is an $\left(q^{2}, q^{2}-1-s^{\prime}, 1\right)$-cover, $q^{2}-1=c^{\prime \prime} s^{\prime \prime}$ and

$$
\left(q^{4}-s\right)\left(q^{2}-1\right)=\left(q^{2}-s^{\prime \prime}\right)\left(q^{4}-1-c^{\prime \prime} s+\frac{c^{\prime \prime}\left(s-s^{\prime \prime}\right)}{\left(s^{\prime \prime}+1\right)}\right) .
$$

If $s^{\prime \prime}=1$, then $c^{\prime \prime}=q^{2}-1$ and $q^{4}-s=q^{4}-1-\left(q^{2}-1\right) s+\left(q^{2}-1\right)(s-1) / 2$, a contradiction. So $s^{\prime \prime}>1$, and

$$
\left(q^{4}-s\right)\left(q^{2}-1\right)>\left(q^{2}-s^{\prime \prime}\right)\left(q^{4}-1-c^{\prime \prime} s+\frac{c^{\prime \prime}\left(s-s^{\prime \prime}\right)}{\left(s^{\prime \prime}+1\right)}\right),
$$

again a contradiction.

Hence, $d=2, Z\left(G_{0}^{(\infty)}\right)$ contains a unique involution $g$ that, for $1 \leq i \leq 2$, maps $e_{i}$ to $-e_{i}, \Delta$ is an $\left(q^{2}, q^{2}-s, 1\right)$-cover and, by Lemma $8, \Omega=\operatorname{Fix}(g)$ is a fibre. This implies $\alpha_{0}(g)=c s-s+1$ and, by Lemma 4 we have

$$
\chi_{1}(g)=\frac{(c s-s)(\sqrt{D}-s / 2)-s+\alpha_{1}(g)}{2 \sqrt{D}} \in \mathbb{Z} .
$$

Suppose $s$ is odd. Then $\alpha_{1}(g)=0$ (otherwise $g$ fixes a vertex in $[a]$ for some $a \in \Omega$, which is impossible) and $c$ is even. In this case the odd number $2 \sqrt{D}$ divides

$$
\sqrt{D} s+s^{2}(c-1) / 2+s=s(\sqrt{D}+s(c-1) / 2+1) .
$$

Since $(s, 2 \sqrt{D})=1,2 \sqrt{D}$ divides $c s-s+2=q^{2}-s+1$. Put $x=(c s+1,2 \sqrt{D})$. By Lemma 3 we have that $x$ equals 1 or is a power of 3 , and, since $(c s-s+2, c s-s)=1$ and $(x, s-2)=1$, $2 \sqrt{D}$ divides $x(c, s-2)$. If $x=1$, then $s-2<2 \sqrt{D} \leq(c, s-2)$, a contradiction. Hence $q^{2}=3^{e}$ and there is an element of order 3 in $G \backslash K$ that has no fixed points. But $(3, s+1)=1$ and, by Lemma 7 we obtain that 3 divides $c s-s+1=q^{2}-s$, which contradicts the fact $(|K|, c s+1)=1$.

Thus $s$ is even and $|K|$ is odd. It follows that $K$ is solvable and $K^{\prime}$ is a normal subgroup of $G$ that is properly contained in $K$. Hence the graph $\Delta^{K^{\prime}}$ admits an arc-transitive action of $G / K^{\prime}$, 
and the abelian group $K / K^{\prime}$ can be considered as the group of all automorphisms of $\Delta^{K^{\prime}}$ fixing its fibres. But the size of a fibre in $\Delta^{K^{\prime}}$ coincides with $\left|K / K^{\prime}\right|$ and $\left(\left|K / K^{\prime}\right|, c s+1\right)=1$, a contradiction to $[9$, Theorem 2.5].

2. Let either $m=p^{2}$ and $p \in\{5,7,11,23,19,29,59\}$, or $m=3^{6}$, or $m=3^{4}$. As it was shown above, $s>2$.

If $m=p^{2}, \mathrm{SL}_{2}(5) \unlhd G_{0}$ and $p \in\{11,19,29,59\}$, then we may assume by Lemma 1 that the triple $(c ; s ; D)$ is one of $(30 ; 4 ; 121),(90 ; 4 ; 361),(210 ; 4 ; 841)$ or $(870 ; 4 ; 3481)$.

If $m=3^{6}=729$ and $\mathrm{SL}_{2}(13) \unlhd G_{0}$, then by Lemma $1(c ; s ; D)=(182 ; 4 ; 729)$.

If $G^{\Sigma}$ is solvable, $m=p^{2}, \mathrm{SL}_{2}(3) \triangleleft G_{0}$ and $p \in\{5,7,11,23\}$, then by Lemma 1 we may assume that the triple $(c ; s ; D)$ is one of $(6 ; 4 ; 25),(12 ; 4 ; 49),(30 ; 4 ; 121)$, or $(132 ; 4 ; 529)$.

If $m=3^{4}=81$ and $G_{0}$ contains a normal extraspecial subgroup $H$ of order 32 , then $(c ; s ; D)=$ $(20 ; 4 ; 81)$.

Since in all these cases $s=4$, there is an $\langle y\rangle$-orbit that is a cycle or $p=5$ and 5 divides $c-1$. Then $|K|=m-4,5$ divides $c\left(c-1, p^{e}\right)$ and $\left|\operatorname{Syl}_{p}(T)\right|=1+l p$ divides $|K|$. It implies $m=p^{2}$ and $p \in\{5,11,19,29,59\}$ or $m=3^{4}$.

Let $m=p^{2}$. We have $p^{2}-4=t(1+l p)$ and $(t ; p)=(1 ; 5)$ (otherwise, $t=t^{\prime} p-4>1$ and $p=t^{\prime}(1+l p)-4 l$, which is impossible). Hence $|K|=21$. If $K$ is cyclic, then by [8, Theorem 9.2], 21 divides $m$, a contradiction. Hence, the subgroup $K^{\prime} \simeq \mathrm{Z}_{7}$ of $K$ is normal in $G$. Then the graph $\Delta^{K^{\prime}}$ admits an arc-transitive action of $G / K^{\prime}$, and $K / K^{\prime}$ can be considered as a group of all automorphisms of $\Delta^{K^{\prime}}$ fixing each its fibre. But $K / K^{\prime} \simeq \mathrm{Z}_{3}$ and hence, by [8, Theorem 9.2], 3 divides $m$, a contradiction.

Let $p=3$. Then $K$ is a cyclic group of order 77 and, by [8, Theorem 9.2], 77 divides $m$, a contradiction.

Thus, the only remaining possibility is $G^{\Sigma} \leq \mathrm{A} L_{1}(q)$. The lemma is proved.

Lemma 14. Suppose $G^{\Sigma} \leq \operatorname{A\Gamma L}_{1}(q)$, where $q=p^{e}$ for a prime $p$. Let $H_{1}$ be the stabiliser of a fibre $F$ in $G^{\Sigma}, H=H_{1} \cap \operatorname{AGL}_{1}(q)$, $\tilde{f}$ and $\tilde{g}$ be two elements of $H$, whose orders are $2^{\prime}$-part and 2-part of $|H|$, respectively, and let $\tilde{z}$ be an involution in $\langle\tilde{g}\rangle$. Denote by $z, f$ and $g$ some representatives of the preimages of the elements $\tilde{z}, \tilde{f}$ and $\tilde{g}$ in $G$, respectively. Then $\operatorname{Fix}(z)$ is a fibre,

$$
\alpha_{0}(z)=q-s, \quad \alpha_{3}(z)=0, \quad \chi_{1}(z)=\left(\alpha_{1}(z)+(\sqrt{D}-s / 2+1)(c s-s)-c s\right) /(2 \sqrt{D})
$$

and the following statements hold.

(1) If $K=1$, then $s$ is even, $s=2$ or cs is divisible by $4,(\sqrt{D}, s) \leq 2$ and $\mid$ fg $\mid$ divides $\alpha_{1}(z)$, and, in particular,

(i) if $s=4$, then $c=d(d+2) / 4, \sqrt{D}=d+1$ for some even integer $d, \alpha_{1}(z)=2\left(p^{e / 2} l+2\right)$, where $l$ is an even integer, and $\alpha_{1}(z)$ is divisible by $(q-1) /(e, q-1)$;

(ii) if $p=3$, then $s+1$ is divisible by 3 .

(2) $G$ acts intransitively on arcs of $\Delta$.

P r o o f. First we show that $\tilde{g} \neq 1$. On the contrary, suppose that $\tilde{g}=1$. Then $|H|$ is odd, $H_{1} / H \leq \mathrm{Z}_{e}$ and since $H_{1}$ is transitive on $\Sigma \backslash\{F\}, e$ is even and $(e)_{2} \geq(q-1)_{2} \geq 4$. If $p-1$ is divisible by 4 , then $\left(p^{e}-1\right)_{2}=(e)_{2}(p-1)_{2}>(e)_{2}$, a contradiction. Hence, $(p-1)_{2}=2$ and the number $p^{2}-1$ is divisible by 4 and divides $p^{e}-1$, and again $\left(p^{e}-1\right)_{2}=(e / 2)_{2}\left(p^{2}-1\right)_{2}>(e)_{2}$, a contradiction. 
(1) Let $K=1$. Let $z, f$ and $g$ denote some representatives of the preimages of $\tilde{z}, \tilde{f}$ and $\tilde{g}$ in $G$, respectively. Then the involution $z \in G_{\{F\}}$ does not fix any fibre from $\Sigma \backslash\{F\}$. If $\operatorname{Fix}(z)=\varnothing$, then $\alpha_{2}(z)=0$ and $\alpha_{1}(z)=c s(c s+1-s)$, that is $z$ fixes an $(s+1)$-clique and by Lemma 7 we have $c s=s+1=2$, a contradiction. It follows by Lemma 8 that $\operatorname{Fix}(z)$ is a fibre, that is $\operatorname{Fix}(z)=F$, $\alpha_{0}(z)=q-s$ and $\alpha_{3}(z)=0$.

Since for each nontrivial element $h \in\langle f g\rangle$ we have $\alpha_{0}(h)+\alpha_{3}(h)=c s-s+1$,

$$
\chi_{1}(h)=\frac{(\sqrt{D}-s / 2+1)\left(\alpha_{0}(h)-1\right)+\alpha_{1}(h)-c s}{2 \sqrt{D}},
$$

hence

$$
\chi_{1}(z)=\frac{(\sqrt{D}-s / 2)(c s-s)+\alpha_{1}(z)-s}{2 \sqrt{D}} .
$$

Suppose $s$ is odd. Then $\alpha_{1}(z)=0$ and $c$ is even. In this case, $\chi_{1}(z) \in \mathbb{Z}$ and the odd number $2 \sqrt{D}$ divides

$$
\sqrt{D} s+s^{2}(c-1) / 2+s=s(\sqrt{D}+s(c-1) / 2+1) .
$$

As $(s, 2 \sqrt{D})=1$, we also get that $2 \sqrt{D}$ divides $c s-s+2=q-s+1$. By repeating the argument from Lemma 13, we obtain that 3 divides $(s-1,2 \sqrt{D}), q=3^{e}$ and there is an element of order 3 in $G$ that has no fixed points. But $(3, s+1)=1$ and, by Lemma 7 we conclude that 3 divides $c s-s+1=q-s$, a contradiction.

Thus, $s$ is even.

If $c s$ is not divisible by 4 , then $c$ is odd. But then

$$
D=c s+(s / 2-1)^{2} \equiv 2 \quad(\bmod 4),
$$

which yields $s=2$. If $s$ is divisible by 4 , then $D$ is odd. Hence $(\sqrt{D}, s) \leq 2$.

As the element $f g$ does not fix any vertex $u$ such that $u^{z} \in[u]$ and centralizes $z$, we get that $|f g|$ divides $\alpha_{1}(z)$.

(1i) Let $s=4$. Then

$$
c=d(d+2) / 4, \quad q=(d+1)^{2} \quad \text { and } \quad \sqrt{D}=d+1 .
$$

Since $\chi_{1}(z) \in \mathbb{Z}, 2(d+1)$ divides $\alpha_{1}(z)-4$ and hence $\alpha_{1}(z)=2\left(p^{e / 2} l+2\right)$ is divisible by $(q-$ 1)/(e,q-1).

Suppose that $\alpha_{1}(z)$ is not divisible by 4 . As $c>2$, we get $\alpha_{1}(z)>0$ and $2(q-1, e)_{2} \geq(q-1)_{2}$. If $p-1$ is divisible by 4 , then $\left(p^{e}-1\right)_{2}=(e)_{2}(p-1)_{2}>2(e)_{2}$, a contradiction. Hence, $(p-1)_{2}=2$ and the number $p^{2}-1$ is divisible by 4 and divides $p^{e}-1$, and

$$
\left(p^{e}-1\right)_{2}=(e / 2)_{2}\left(p^{2}-1\right)_{2}>2(e)_{2},
$$

a contradiction. Thus $l$ is even.

(1ii) If $y$ is an element of order $p$ of the socle of $G$, then $\alpha_{i}(y)=0$ for $i=0,3$ and $\chi_{1}(y)=$ $\left(\alpha_{1}(y)-q\right) /(2 \sqrt{D})$. In the case $p=3$ in view of Lemma 7 we conclude that 3 divides $s+1$.

(2) Suppose that $G$ acts transitively on $\operatorname{arcs}$ of $\Delta$. Then by Lemma 12 we have $|K|=c s-s+1$ and $G_{a} \simeq G_{\{F\}} / K(a \in F)$. Let $z, f$ and $g$ denote some representatives of the preimages of the elements $\tilde{z}, \tilde{f}$ and $\tilde{g}$ in $G_{a}$. Then we may assume that $z$ is an involution and it does not fix any fibre from $\Sigma \backslash\{F\}$. Hence by Lemma $8, \operatorname{Fix}(z)$ is a fibre, $\alpha_{0}(z)=q-s$ and $\alpha_{3}(z)=0$.

Since for each nontrivial element $h \in\langle f g\rangle$ we have $\alpha_{0}(h)+\alpha_{3}(h)=c s-s+1$,

$$
\chi_{1}(z)=\frac{(\sqrt{D}-s / 2)(c s-s)+\alpha_{1}(z)-s}{2 \sqrt{D}} .
$$


Suppose $s$ is odd. Then $\alpha_{1}(z)=0$ and $c$ is even. In this case, $\chi_{1}(z) \in \mathbb{Z}$, and the odd number $2 \sqrt{D}$ divides

$$
\sqrt{D} s+s^{2}(c-1) / 2+s=s(\sqrt{D}+s(c-1) / 2+1) .
$$

Again, by repeating the argument from Lemma 13, we obtain a contradiction to Lemma 7 .

Thus $s$ is even and $|K|$ is odd. It follows that $K$ is solvable and $K^{\prime}<K$. Hence the graph $\Delta^{K^{\prime}}$ is a non-bipartite antipodal distance-regular graph of diameter 3 that admits an arc-transitive action of $G / K^{\prime}$, and the abelian group $K / K^{\prime}$ can be considered as the group of all automorphisms of $\Delta^{K^{\prime}}$ fixing its fibres. But the size of a fibre in $\Delta^{K^{\prime}}$ coincides with $\left|K / K^{\prime}\right|$ and $\left(\left|K / K^{\prime}\right|, c s+1\right)=1$, a contradiction to [9, Theorem 2.5]. The lemma is proved.

P r o o f of Theorem 1 follows immediately from Lemmas 10, 11, 13 and 14.

P r o o f of Theorem 2. Assume $\Delta$ is not a 6-cycle. First note that each edge-transitive group of automorphisms of $\Delta$ induces a 2-homogeneous permutation group on $\Sigma$. It is also clear that if there is an edge-transitive group of automorphisms of $\Delta$, then it acts transitively on its vertices as well, because $\Delta$ is a non-bipartite graph whenever $(c ; s) \neq(2 ; 1)$. The case $(c ; s)=(56 ; 1)$ cannot occur, as otherwise the order of $G$ would be divisible by $57 \cdot 56$, which is impossible by [17] (see also [13]). Thus, it remains to apply Theorem 1.

\section{Open Questions}

We conclude with few open questions.

1. Is there a half-transitive $(n, r, 1)$-cover?

2. Is there any $(n, r, 1)$-cover with $n-r>1$ that possesses a group of automorphisms acting 2-homogeneously on the fibres when $n-1$ is not a power of 2 or $n-r>2$ ?

\section{Acknowledgements}

The author thanks A.A. Makhnev and A.L. Gavrilyuk for discussions and comments on earlier versions of the manuscript.

\section{REFERENCES}

1. Aschbacher M. Finite Group Theory, 2nd ed. Cambridge: Cambridge University Press, 2000. 305 p. DOI: 10.1017/CBO9781139175319

2. Blokhuis A., Brouwer A. E. Geodetic graphs of diameter two. Geom. Dedicata, 1988. Vol. 25. P. 527-533. DOI: $10.1007 / \mathrm{BF} 00191941$

3. Brouwer A E., Cohen A. M., Neumaier A. Distance-Regular Graphs. Berlin etc: Springer-Verlag, 1989. 494 p. DOI: $10.1007 / 978-3-642-74341-2$

4. Cameron P.J. Permutation Groups. Cambridge: Cambridge Univ. Press, 1999. 220 p. DOI: $10.1017 /$ CBO9780511623677

5. Gardiner A. Antipodal covering graphs. J. Comb. Theory B., 1974. Vol. 16, No. 3. P. 255-273. DOI: 10.1016/0095-8956(74)90072-0

6. Gavrilyuk A.L., Makhnev A.A. Geodesic graphs with homogeneity conditions. Dokl. Math., 2008. Vol. 78. P. 743-745. DOI: 10.1134/S1064562408050268

7. Godsil C.D. Covers of complete graphs. Adv. Stud. Pure Math., 1996. Vol. 24. P. 137-163. DOI: $10.2969 / \mathrm{aspm} / 02410137$ 
8. Godsil C. D., Hensel A. D. Distance regular covers of the complete graph. J. Combin. Theory Ser. B., 1992. Vol. 56. P. 205-238. DOI: 10.1016/0095-8956(92)90019-T

9. Godsil C. D., Liebler R. A., Praeger C. E. Antipodal distance transitive covers of complete graphs. Europ. J. Comb., 1998. Vol. 19, No. 4. P. 455-478. DOI: 10.1006/eujc.1997.0190

10. Hoffman A. J., Singleton R. R. Moore graphs with diameter 2 and 3. IEEE Xplore. IBM J. of Research and Development, 1960. Vol. 5, No. 4. P. 497-504. DOI: 10.1147/rd.45.0497

11. Kantor W. M. k-homogeneous groups. Math. Z., 1972. Vol. 124. P. 261-265. DOI: 10.1007/BF01113919

12. Kantor W. M. Moore geometries and rank 3 groups having $\mu=1$. Q. J. Math., 1977. Vol. 28 , No. 3. P. 309-328. DOI: 10.1093/qmath/28.3.309

13. Mačaj M., Šráň J. Search for properties of the missing Moore graph. Linear Algebra Appl., 2010. Vol. 432, No. 9. P. 2381-2398. DOI: 10.1016/j.laa.2009.07.018

14. Makhnev A.A., Paduchikh D. V., Tsiovkina L. Yu. Edge-symmetric distance-regular coverings of complete graphs: the almost simple case. Algebra Logic, 2018. Vol. 57, No. 2. P. 141-152. DOI: $10.1007 / \mathrm{s} 10469-018-9486-5$

15. Makhnev A. A., Tsiovkina L. Yu. Arc-transitive antipodal distance-regular graphs of diameter three related to $P S L_{d}(q)$. Sib. Elektron. Mat. Izv., 2016. Vol. 13. P. 1339-1345. DOI: 10.17377/semi.2016.13.104

16. Makhnev A. A., Tsiovkina L. Yu. Antipodal Distance-Regular Graphs and Their Automorphisms. Novosibirsk: Sobolev Institute of Mathematics Publishing House, 2018. 196 p. (in Russian)

17. Makhnev A. A., Paduchikh D. V. Automorphisms of Aschbacher graphs. Algebra Logic, 2001. Vol. 40, No. 2. P. 69-74. DOI: 10.1023/A:1010217919915

18. Mazurov V. D. Minimal permutation representations of finite simple classical groups. Special linear, symplectic, and unitary groups. Algebra Logic, 1993. Vol. 32, No. 3. P. 142-153. DOI: 10.1007/BF02261693

19. Tsiovkina L. Yu. Two new infinite families of arc-transitive antipodal distance-regular graphs of diameter three with $\lambda=\mu$ related to groups $S z(q)$ and ${ }^{2} G_{2}(q)$. J. Algebr. Comb., 2015. Vol. 41, No. 4. P. 10791087. DOI: $10.1007 / \mathrm{s} 10801-014-0566-\mathrm{x}$

20. Tsiovkina L. Yu. Arc-transitive antipodal distance-regular covers of complete graphs related to $S U_{3}(q)$. Discrete Math., 2017. Vol. 340, No. 2. P. 63-71. DOI: 10.1016/j.disc.2016.08.001

21. Tsiovkina L. Yu. On affine distance-regular covers of complete graphs. Sib. Elektron. Mat. Izv., 2015. Vol. 12. P. 998-1005. (in Russian) DOI: 10.17377/semi.2015.12.086

22. Tsiovkina L. Yu. Arc-transitive groups of automorphisms of antipodal distance-regular graphs of diameter 3 in affine case. Sib. Elektron. Mat. Izv., 2020. Vol. 17. P. 445-495. (in Russian) DOI: $10.33048 /$ semi.2020.17.029 